
Not for reproduction, distribution or commercial use.

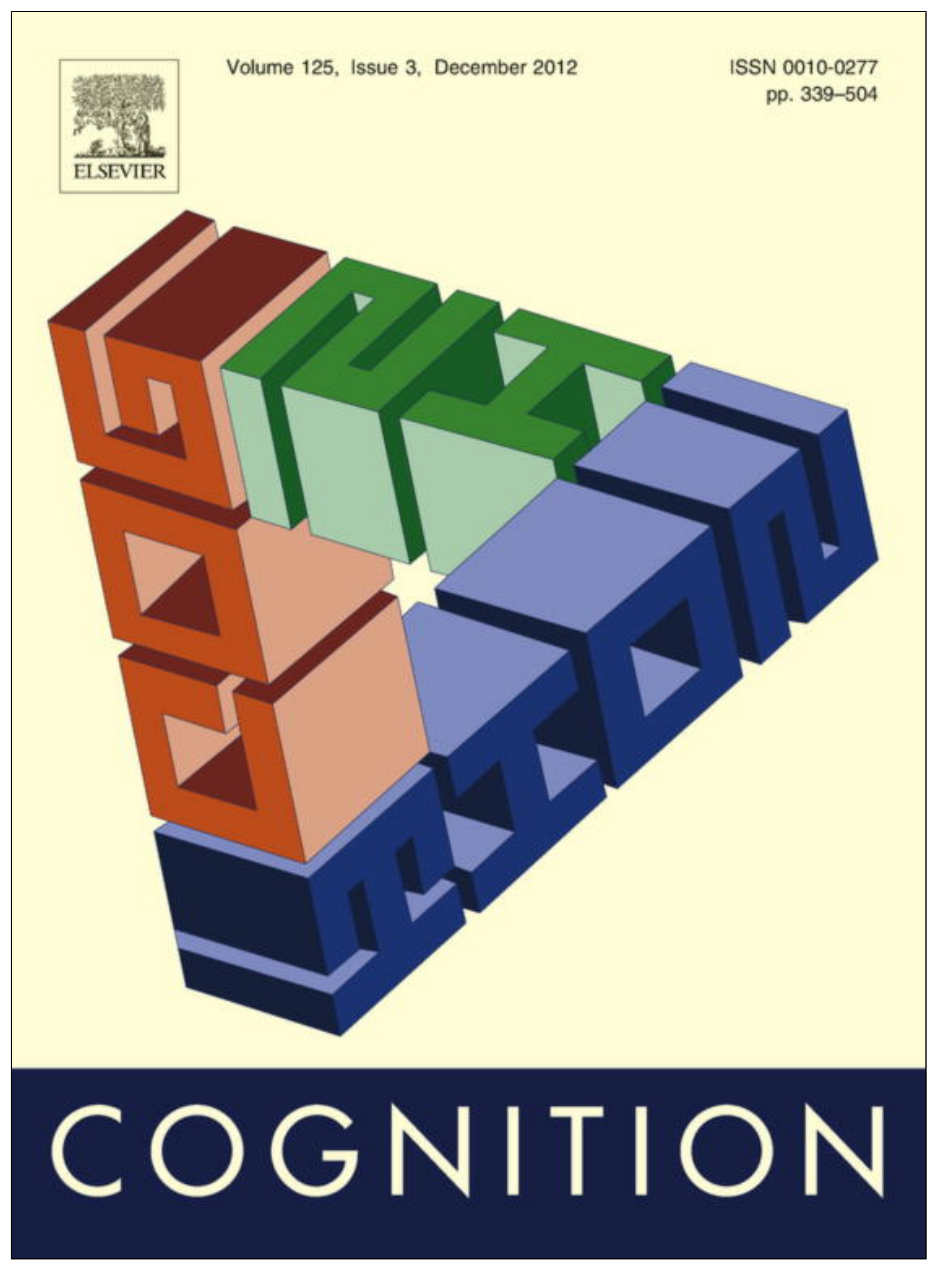

This article appeared in a journal published by Elsevier. The attached copy is furnished to the author for internal non-commercial research and education use, including for instruction at the authors institution and sharing with colleagues.

Other uses, including reproduction and distribution, or selling or licensing copies, or posting to personal, institutional or third party websites are prohibited.

In most cases authors are permitted to post their version of the article (e.g. in Word or Tex form) to their personal website or institutional repository. Authors requiring further information regarding Elsevier's archiving and manuscript policies are encouraged to visit:

http://www.elsevier.com/copyright 


\title{
Picasso in the mind's eye of the beholder: Three-dimensional filling-in of ambiguous line drawings
}

\author{
Jan Koenderink ${ }^{\mathrm{a}, \mathrm{b}}$, Andrea van Doorn ${ }^{\mathrm{c}}$, Johan Wagemans ${ }^{\mathrm{a}, *}$ \\ ${ }^{a}$ University of Leuven (KU Leuven), Laboratory of Experimental Psychology, Belgium \\ ${ }^{\mathrm{b}}$ Delft University of Technology, Electrical Engineering, Mathematics, and Computer Science, The Netherlands \\ ${ }^{c}$ Delft University of Technology, Industrial Design, The Netherlands
}

\section{A R T I C L E I N F O}

\section{Article history:}

Received 26 December 2011

Revised 26 July 2012

Accepted 27 July 2012

Available online 28 August 2012

\section{Keywords:}

Shape perception

Perceptual filling-in

Ambiguous figures

Pictorial relief

Depth perception

Art perception

\begin{abstract}
A B S T R A C T
Cartoon-style line drawings contain very condensed information, after all most of the page stays blank. Yet, they constrain the contents of immediate visual awareness to an extraordinary extent. This is true even for drawings that are - though nominally "representational" - not even in central projection. Moreover, the strokes used in a drawing may stand for a bewildering variety of entities in the world. We studied Picasso drawings from the 1940s in which the artist famously combined multiple viewpoints. We find that the pictorial reliefs obtained from various observers agree to a large extent, and that the differences are of a very specific nature, typically involving variations in the mutual spatial attitudes of rigid body parts in figure studies. Although the purely planar layout of the drawings accounts for much of visual awareness, observers also use the sparse depth cues provided by the artist to evoke volumetric impressions. Observers also freely insert "template knowledge" about the structure of familiar generic objects.
\end{abstract}

(C) 2012 Elsevier B.V. All rights reserved.

\section{Introduction}

Cartoon-like line drawings are apparently easy to understand, even by the public with no expertise in the visual arts (Arnheim, 1974; Kennedy \& Silver, 1974; Koenderink, van Doorn, Cristou, \& Lappin, 1996a). We say “apparently”, because there appears to be no records of specific inability to understand line drawings with intact ability to understand photographs. This is perhaps surprising in view of the fact that in a cartoon-like rendering most of the picture plane remains blank, whereas the lines may stand for a bewildering variety of mutually categorically different properties. Nor do such scenes need to exist at all, or to be physically realizable. Pictorial worlds may deviate greatly from the natural environments as depicted in holiday snapshot photographs say.

\footnotetext{
* Corresponding author. Address: University of Leuven (KU Leuven), Laboratory of Experimental Psychology, Tiensestraat 102, Box 3711, BE3000 Leuven, Belgium. Tel.: +32 163259 69; fax: +32 16326099 .

E-mail address: Johan.Wagemans@psy.kuleuven.be (J. Wagemans).
}

It is hard to conceive of algorithmic or physiological processes that might "fill-in the blanks" in line drawings, especially when the drawings are not from a central viewpoint (thus could not be derived from photographs). Yet, visual awareness arises spontaneously, and evidently outside the realm of reflective thought. This is an aspect of mental processes, that might perhaps be denoted precognitive.

Since a stroke may stand for so many different entities (Fig. 1, left; Steinberg, 2011), and may even change its intended meaning along its course, the reading of a drawing necessarily involves the whole drawing, or at least a significant part of it. Parsing the drawing on a local basis is not likely to carry the observer very far. Yet, even short strokes often induce meaning by themselves. For instance, a short but highly curved segment leads to the awareness of an inside-outside relationship, that is to say, an object. The "stuff" is felt to be at the concave side of a curvilinear arc. Such partial meanings are necessarily ambiguous (e.g., an oval stroke may stand for an object, as well as for a hole), and are easily overridden by context. Strokes 

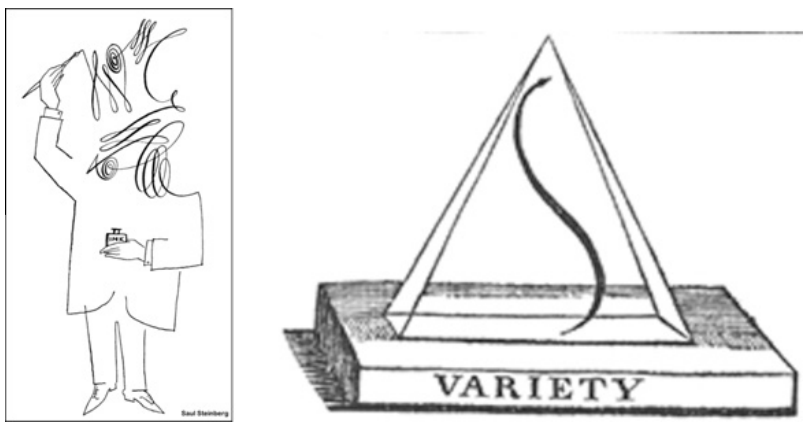

Fig. 1. Left: A drawing by Paul Steinberg (1914-1999) (Steinberg, 2011), illustrating the use of strokes with a wide variety of "meaning". Notice the meaning of some strokes varying along their course. Right: William Hogarth's "line of beauty" from the front page of his book "The Analysis of Beauty" of 1773 .

may also have an abstract, purely esthetic "meaning", like Hogarth's (1773) "line of beauty" (Fig. 1, right).

Many line drawings are mainly perceived as "flat", even if their intended interpretation is a three-dimensional (3D) scene (Crane, 1900). Planar configurations often stand as symbolic for 3-D entities. For instance, a circle may stand for a sphere, and a square for a cube, without implication of solidity. In many cases it may be hard to decide whether a drawing is an essentially planar design, or the rendering of entities. However, there is little doubt that at least some drawings aim at a subsidiary awareness of substantial volume. Of course, visual artists always care for good planar compositions too, even in realistic renderings of volumetric scenes (Hildebrand, 1893).

In case a line drawing appears to depict a solid, smooth object, much of the blank area of the picture plane transforms into a curved surface (a pictorial relief) in "pictorial space". Since there "is nothing" to go on inside this area, such reliefs are evidently constructions of the mind (Koenderink et al., 1996a). The drawing somehow constrains the microgenesis (Brown, 1996) of the observer. ${ }^{1}$ Given the scarcity of data, one infers that such reliefs are to some degree idiosyncratic.

This defines the main goal of this study. We measure the pictorial reliefs of a number of observers viewing the same cartoon-like line drawings. We study the nature of the differences between observers, or a single observer at a number of - hopefully largely independent - occasions.

\subsection{The potential meaning of lines}

There have been various attempts to list the potential "meanings" of strokes (Crane, 1900; Hanks \& Belliston, 1992; Mellem, 2009; Speed, 1917). ${ }^{2}$ A stroke as such is meaningless, but its author may well have had an articulate intention (Brentano, 1874). The observer likewise has inten-

\footnotetext{
1 We use the conventional term "microgenesis" for the unconscious process that generates immediate visual awareness. Perhaps "iconogenesis" would be a better term, but it is not in common use.

2 Instead of "stroke" one often uses "line". With "lines" one does not just mean the Euclidean "straight line", but also longish and smooth curves. This is the meaning of "line" usually used in the visual arts. It is the way we use the term in this paper.
}

tions (in Brentano's, 1874 sense), namely to let the line fit his or hers immediate visual awareness.

Lines in the geometrical sense have only structure, not meaning. The meaning exists relative to the author or the reader, and these are generally different meanings, although they may agree to a certain extent.

It is not necessarily the case that the author is fully aware of the meaning of all lines, even if he or she actually draws them over time. The author has at least a subsidiary awareness in the process of drawing though. The reader is necessarily confronted with all lines simultaneously. For instance, since many lines partake in the suggestion of solid objects, it is often the case that lines that are at some distance in the drawing, and not even mutually connected, should be read as a single glyph.

Although strokes may stand for many things (Crane, 1900; Hanks \& Belliston, 1992; Kennedy \& Silver, 1974), there are also various instances where they are not drawn. For example, both segmentation and edge finding are common algorithms applied in image processing that result in potential strokes, or collections of line elements. Yet, the majority of lines generated by such methods will never be used by the visual artist. Obvious examples are the outlines of cast shadows (salient results from edge finding algorithms; see Fig. 2, left) and certain isophotes (like the partitions of the blue sky found by most segmentation algorithms; see Fig. 2, right). Apparently, visual artists pick and choose between "objectively available" linear elements in a scene.

\subsection{Attempts to define meaningful lines}

Attempts to define the meaning of lines may be divided roughly into two categorically different types. One has to do with the expressive use of lines, the other with the function of lines to indicate aspects of 3-D geometrical properties.

The work on expressive properties has remained fairly restricted, at least in science. It has mainly been focused on such properties as sharp raggedness versus articulated smoothness. The former tends to be associated with active, aggressive, and male natures, the latter with passive, female natures (the "bouba-kiki effect", see Köhler, 1929; Maurer, Pathman, \& Mondloch, 2006). Much more elaborate work can be found in the literature of the visual arts, for instance in Klee's (1956) notebooks, or Wassily Kandinsky's Punkt und Linie zu Fläche (1926).

The work on geometrical indication of lines has led to a large literature in the sciences. It is associated with the "shape from contour" problems, and with the computer graphics methods of artistic rendering (Cole et al., 2009; DeCarlo, Finkelstein, Rusinkiewicz, \& Santella, 2003; Forsyth \& Ponce, 2002; Judd, Durand, \& Adelson, 2007; Koenderink, 1990; Koenderink \& van Doorn, 1982; Marr, 1982; Poggio, 1984). Initial work has been focused on "shape from contour", and authors have suggested that human observers interpret lines as certain classes of surface markings, for instance geodesics, or lines of principal curvature (Hoffman, 1983; Stevens, 1981). This may then be interpreted as a valid "inverse optics" problem (Poggio, 1984). 

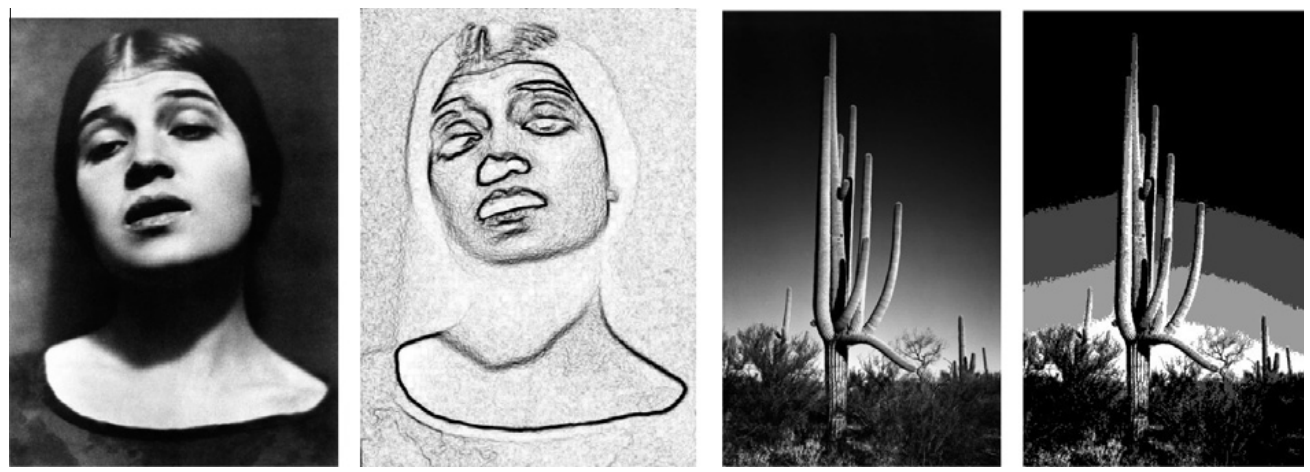

Fig. 2. Left: Two images: A photograph by Edward Weston (1886-1958) (Weston, 2011) and the result of an "edge finder". Notice the edge outlining the cast shadow of the chin. Right: Two images: A photograph by Ansel Adams (1902-1984) (Adams, 2011) and a segmented version. Notice the segmentation of the sky. Edge finding would produce curved lines in the sky. In either case the image processing algorithms yield "potential strokes" such as an artist would never use. The reader would have difficulties making sense of such strokes; it would be confusing rather than informative.

Later work exploited the importance of genericity (Hoffman, 1998; Thom, 1973). This would allow almost certain inferences by way of a singular (probabilities zero or one) application of Bayes' theorem. Examples are the "blocks world" (Winograd, 1972), and the contour singularities like the ending contour or the T-junctions (Koenderink, 1990; Koenderink \& van Doorn, 1982, 1998).

More recently, computer graphics has developed methods to generate lines indicative of certain surface topographical landmarks, such as ruts or ridges (DeCarlo et al., 2003; Koenderink, 1990; Koenderink \& van Doorn, 1998).

All such methods are developed in the context of sparse, but essentially "veridical" rendering. That is to say, all pictures are in perfect central perspective. (That does not necessarily mean "linear perspective", but in any case "seen from a single viewpoint".) From this viewpoint, line drawings are much like photographs in the way they are related to their 3-D prototypes. It is mainly in the extreme sparseness of the structure of the rendering that they are anything special.

\subsection{Types of line drawings}

Line drawings exist in a bewildering variety. Many are typical for very specific professional use. Examples are scientific diagrams and graphs, cartographic renderings of various kinds, architectural drawings, instructional drawings intended for manuals and public address, and so forth. An entirely different category is that of the artistic renderings. In this paper we only touch on the case of artistic renderings in a "representational" mode.

Note that "representational mode" does not imply what is generally considered as "realistic". It is not at all obvious how to define "representational" or "realistic" art, but, at least for the purpose of this paper, one defining characteristic is a unitary view. That is to say, the picture is topographically isomorphic to the "optic array" (Gibson, 1950) for some unique viewpoint (Pirenne, 1970). Here "optic array" means the space of simultaneous visual directions (Euclid, ca. 300 BC). This space has (potentially at least) the topology of a sphere, although typically only some convex spherical patch is being used (Koenderink \& van Doorn, 2003).
From an optical perspective, the scene in front of the observer is generally composed of opaque volumetric objects. For the sake of conciseness we may suppose these to be bounded by piece-wise smooth surfaces. A spherical object is bounded by a smooth surface, whereas a cubical object is bounded by a surface made up of six smooth pieces (of which at most three are visible, or, if the strict notion of a "cube" is relaxed, at most five), connected at "edges". Either surface may be used to stand for the "surface of a treetop" as the numerous books on "how to draw" reveal. That is to say, the artist tends to see piecewise smooth surfaces all over the place (Crane, 1900; Hamm, 1963; Hanks \& Belliston, 1992).

The surfaces of opaque solid objects map on patches in the optic array (Gibson, 1950). The boundaries of these patches can be of different natures. The most important are:

- The "contour," that is the projection of the "rim," which is a curve on the surface that divides it into visible and not visible (or "occluded") parts. On a smooth surface the rim is a smooth curve, but the contour may have certain salient features, namely "T-junctions" and "terminations" (see below).

- The "cutting edges" (Hanks \& Belliston, 1992), which are the projections of edges on the surface that are also part of the rim.

In case the object is not volumetric, but a curvilinear sheet, there is often another type, the "flag edge." A flag edge is the projection of the visible edge of the sheet.

In case the object is not even extended in two dimensions, but a mere "wire," the projection of the wire itself is relevant. The generic case is the opaque volumetric object.

A computer graphics rendering in mere line would certainly show the contours, cutting edges, flag edges, and wire projections in full. This might be called a "complete cartoon rendering" of the scene.

Apart from these lines, there is a variety of other geometrical entities that might be rendered. For instance, the edges on the surface that are not simultaneously part of the rim are also informative, they are like singular "(mountain) ridges". Drawing their projection makes all 
the difference when one delineates a polyhedron. (Think of a cube seen from the body diagonal, and drawn as a regular hexagon.) Such projections are not "cutting edges", but are usually added (e.g., in thinner line) in "complete cartoon renderings" (Hanks \& Belliston, 1992). Something similar applies to smooth surfaces (DeCarlo et al., 2003; Koenderink, 1990; Koenderink \& van Doorn, 1998). Certain loci of extremal curvature are known as "ridges", "ruts", "crests", and so forth (Koenderink, 1990). Their projections are informative, when not confused with edges or contours proper.

An artistic rendering will rarely be exactly like such a geometrically defined graphics rendering. Parts of the lines will be omitted, sometimes parts of different pieces will be joined. Typically, the drawing will deviate from linear perspective (or other well-defined ways to map the optic array on paper) in significant ways. Often, various salient details like T-junctions, contour terminations, vertices (projections of points where edges meet) will receive special treatment. One reason to do this is to increase the likelihood that the drawing will be read as a scene in depth, another - conflicting - reason is to emphasize the nature of lines as a flat configuration, the "arabesque" and pattern properties. In artistic drawing those competing aspects are somehow brought into harmony. That is perhaps the major reason why artistic drawings rarely resemble complete cartoon renderings. The latter variety tends to look more fit for children's coloring books than for the walls of an art gallery (Fig. 3).

\subsection{The choice of stimuli}

We decided on the use of artistic renderings of the "representational" variety. The drawings are not meant as mere arabesques or pleasant filling of areas (like hatches, or scribbled "texture"), but suggest readily recognizable scenes, such as pastoral scenes, erotic anecdotes, and so forth (Gombrich, 1961). They need not present actual scenes, or even physically possible ones (Gurney, 1958). "Representational" does in no way imply that inverse optics would be a feasible way of visually parsing the drawing. For one thing, such drawings need not necessarily be
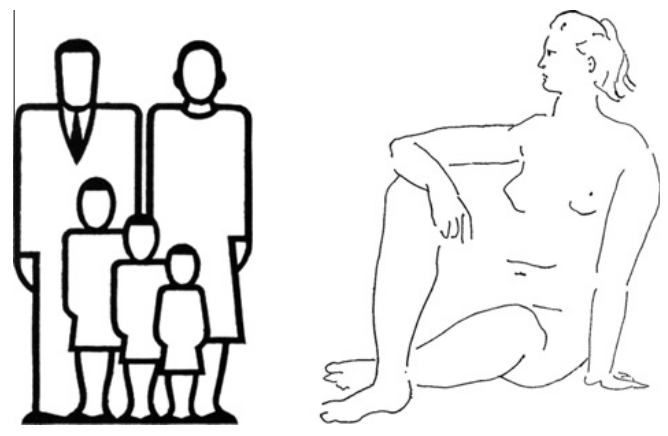

Fig. 3. Left: One of Neurath's (1936) ISOTYPE glyphs. It is fit for a children's coloring book. Note how all regions are perfectly outlined. (All this is by design, of course.) Right: A drawing by Picasso. Much of the contour "is lacking" in the Picasso drawing. The inside-outside relation is ambiguous, and the drawing would hardly classify for the regular coloring book. Notice that the outer limits are clearly delineated and almost form a perfect square: This is a strong planar composition. central projections (perspectives) of scenes, nor necessarily arbitrary deformations of these. A drawing might possibly represent a figure as seen from all sides simultaneously, evidently not a possibility provided by any variety of central projection.

Such drawings are common enough in the visual arts of the West from the twentieth century on. For instance, Pablo Picasso provided examples galore (Berger, 1989). During the 1940s Picasso explored the expressive possibilities of multiple perspectives in a single drawing. These drawings are of interest to us because there is evidently no "ground truth" of the matter. The drawings depict easily recognizable entities, such as human figures, but in ways such as nobody could have experienced before. This is of immediate interest to our quest. Observers are required to construct pictorial reliefs for which they cannot be supposed to possess global templates, yet the drawings constrain the microgenesis significantly.

Many of Picasso's drawings from this period are so extreme (certainly as judged in the context of the era in which they were produced) that it might be doubted whereas observers might be able to arrive at 3-D pictorial presentations. Of course, it would be perfectly feasible that observers might enjoy these drawings as two-dimensional designs with mere symbolic reference to 3-D entities. That would place them in the category of "design", putting them in the realm of heraldry, abstract embroidery, and so forth.

For these images there is no such a thing as "correct viewpoint", or "ground truth". It is not possible to know Picasso's intentions in retrospect. Even during his lifetime, or right after the drawing session, that would have been largely impossible. All one can do is record what observers experience when confronted with the work.

We selected three drawings by Pablo Picasso, ranging from passable as "representational" to evidently impossible as a view from a single viewpoint. The drawings were scanned from a book compilation of Picasso drawings (Bouret, 1950), the lines set to high contrast in Photoshop, and pertinent regions cut out. Discarding contextual detail was necessary in order to maximize screen estate. That was considered acceptable because the discarded detail was irrelevant to the construction of the intended pictorial relief.

The three stimuli are shown in Fig. 4. Notice that the drawings contain material that was not used in the experiment. For instance, the man lying on his belly in Stimulus I, Venus' dove in Stimulus II, or the flying Amor in Stimulus III were not part of the measurement area.

\section{Methods}

\subsection{Participants}

Five observers $\{A D, H R, J K, J W, F H\}$ participated in the experiment, three of them the authors (that are $\{A D, J K$, JW $\}$ ). Four observers $\{A D, H R, J K, J W\}$ ran the experiment three times each. All except $\{\mathrm{JW}, \mathrm{FH}\}$ were familiar with the task, though only two (that are $\{A D, J K\}$ ) had explicitly studied Picasso drawings from this particular period. Distribution over genders was roughly equal ( $\{\mathrm{HR}, \mathrm{JK}, \mathrm{JW}\}$ male, $\{A D, F H\}$ female). Ages ranged from 30 s to 60 s. 
Stimulus I

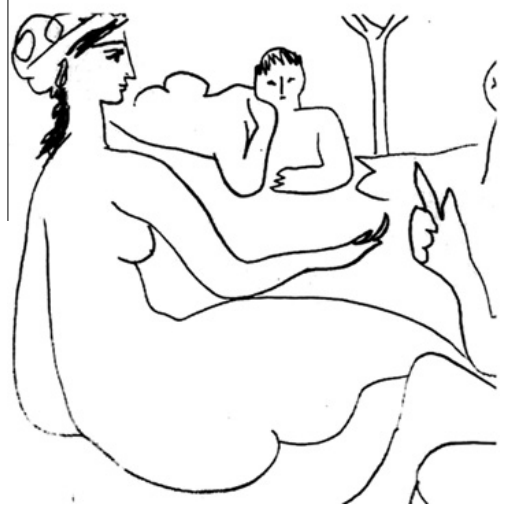

Stimulus II

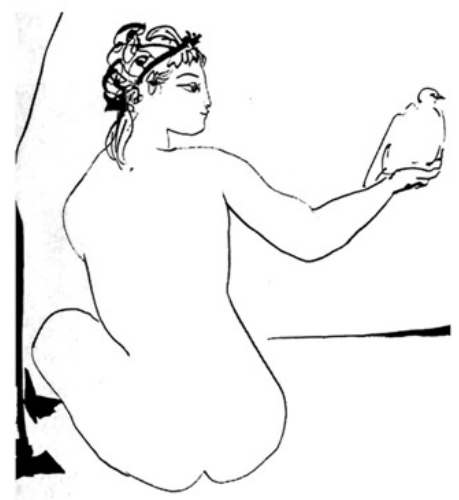

Stimulus III

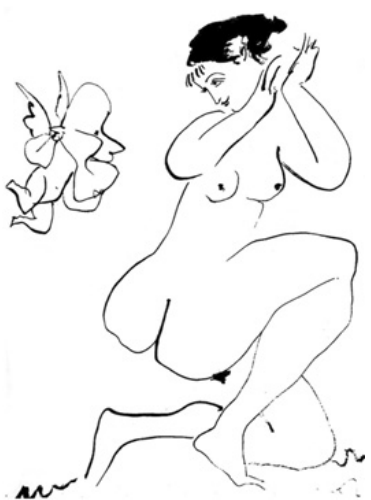

Fig. 4. The three Picasso drawings used in the experiment (from left to right: Stimulus I, II, and III). Only part of the torsos was actually used, in particular the outer extremities were not used.
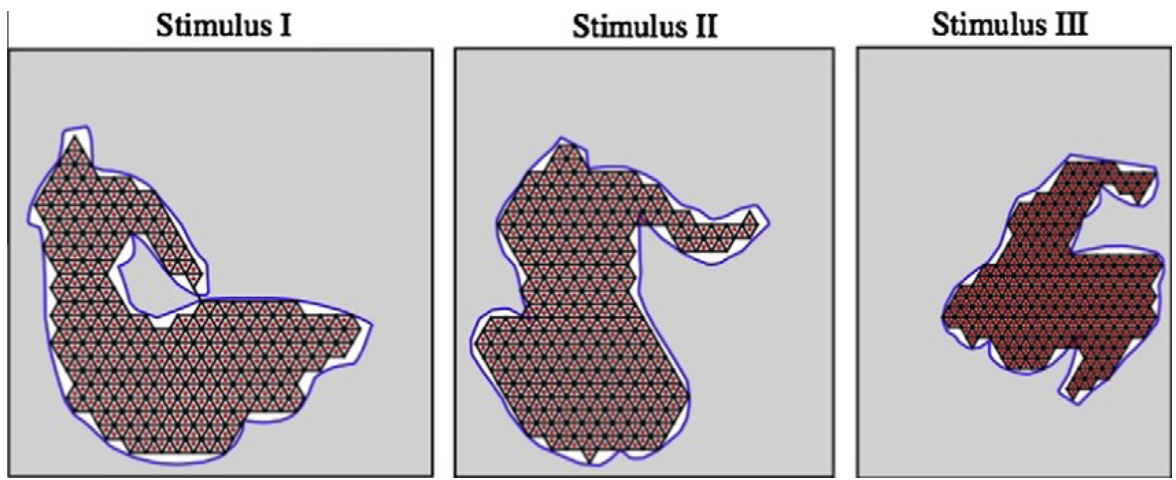

Fig. 5. The triangulations of the three stimuli. The red dots are at the barycenters of the faces ( 422 for Stimulus I, 431 for Stimulus II, and 424 for Stimulus III). The rectangles conform to the actual cut-outs used in the experiment, allowing one to judge how the measurement areas fitted in the picture frame. (In subsequent figures, we will frequently crop to the significant areas.).

\subsection{Research design}

In the stimulus drawings (Fig. 4) we traced the outline of the measurement areas and automatically generated 256 points (uniformly distributed in a regular triangular lattice) in the interiors. Apart from these vertices, we also computed the faces and the barycenters of the faces for these triangulations. Notice that there are many more faces than vertices, see Fig. 5 .

The method of determining the spatial attitude at a point is that of adjusting an elliptical "gauge figure" (Fig. 6) so as to appear as "a circle painted on the surface", appearing in foreshortening due to non-frontal spatial attitude. We have described it before (Koenderink \& van Doorn, 1995, 2003; Koenderink, van Doorn, \& Kappers, 1992, 1996b; Koenderink et al., 1996a).

The observers never saw the triangulations directly, but the measurements were done at the barycenters of the triangulation, in random order, one at a time. It is unlikely that any naive observer would spontaneously notice that the points at which measurements were taken were located on a triangular grid. Observers sometimes complained locations were repeated, which was not the case.

Notice that the spatial attitude of the local pictorial relief is defined as the fiducial attitude (slant and tilt used in the generation of the graphics of the gauge figure). This is

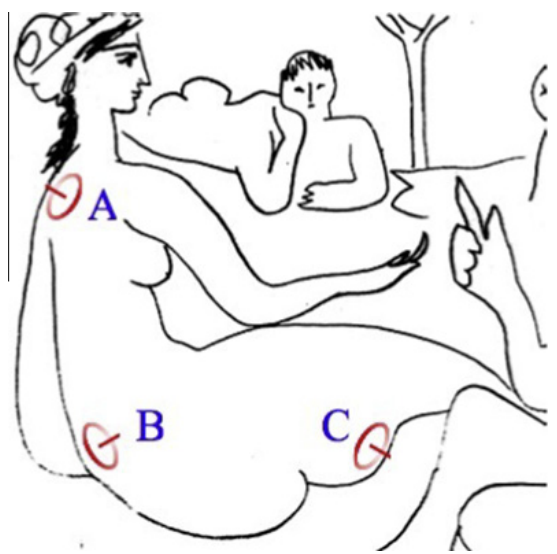

Fig. 6. The elliptical "gauge figure" is drawn as the wireframe rendering of a circular wheel with an axle sticking out at right angles, in orthographic projection (three instances $\mathrm{A}, \mathrm{B}$, and $\mathrm{C}$ shown). The length of the axle equals the radius of the wheel. Notice that the elliptical area is oriented (cannot be perceptually "inverted") because the axle is seen to "stick out". The obliqueness of the disk is defined as the "slant" angle, the direction of slanting as the "tilt" angle. The slant ranges from $0^{\circ}$ (frontal view) to $90^{\circ}$ (edge on view), whereas the tilt runs over the full $360^{\circ}$. The tilt needs to be referred to a reference (the horizontal to the right, anticlockwise orientation). The three gauge figures have been placed on Stimulus I (in the experiment never more than a single gauge figure is visible at any time). Of these " $A$ " and " $C$ " might perhaps be seen to fit the relief, whereas most observers would agree that "B" does not fit at all. 
similar to the classical way of defining "length" as the indication on a divided ruler that is brought into coincidence with the extent to be measured, or "weight" as the sum of standard weights that together balance the object to be measured on a conventional pair of scales. The fact that the method is an operational definition of spatial attitude is crucial. Pictorial spatial attitude is not an entity that exists prior and apart from the measurement. This is a fundamental issue that is only too often misunderstood.

As the final result of a session we obtain the depths at the vertices, and measure of consistency at any vertex. All further analyzes are based on these data.

The depth values obtained by this method have no natural origin. We simply (and arbitrarily) set the mean of the depths at all vertices to zero. That depth has no natural origin makes intuitive sense, because the eye is not itself located in pictorial space. The familiar concept of "distance to the eye" (or "range") is not applicable to pictorial space, as it is in physical space.

Moreover, we have found that observers often commit "mental changes of viewpoint" in which they change the overall attitudes of pictorial reliefs. They also frequently change their depth scale, either dilating, or contracting it. Such changes have been described by artists (Hildebrand, 1893), and have been found to account for much (often most) of the variation between observers confronted with the same picture (Cornelis, van Doorn, \& de Ridder, 2003; Cornelis, van Doorn, \& Wagemans, 2009; Koenderink, van Doorn, Kappers, \& Todd, 2001). Thus, such idiosyncratic transformations are an important empirical fact, and we find it essential to take them into account in our analysis. More recently, we have also identified the formal reasons for this (Koenderink \& van Doorn, 2008), and have formulated a fairly complete (non-Euclidean) geometry of pictorial space (Koenderink \& van Doorn, in press). In the present experiment, we freely use such geometrical methods where required; for details on the methods the reader is referred to our previous papers.

\subsection{Procedure}

The stimulus was presented on a DELL U2410f monitor, $1920 \times 1200$ pixels LCD screen. The viewing distance was $78 \mathrm{~cm}$. Viewing was monocular with the dominant eye, the other eye being patched or closed. Viewing was through a $4 \mathrm{~cm}$ circular aperture at fixed position, the head being stabilized by a chin and forehead rest. The pictures subtended about $30-40^{\circ}$ of visual angle, thus the foreshortening factor at the left and right edges was about 0.95 , within $5 \%$ from unity, which was our design objective. The room was darkened, all illumination being due to the screen. The walls of the experimental room were painted black.

Interaction took place via a standard computer mouse, whose position within a circular area controlled the slant and tilt of the gauge figure. All observers considered the task a "natural" one.

The observers visited all barycenters of the triangulation, one after the other, in random order. When they were satisfied with the spatial attitude of the gauge figure they hit the space bar on the keyboard, which triggered the next position. All barycenters were visited in a single session. Observers finished a session in less than an hour.

\section{Results}

\subsection{Typical results for a single observer, single session}

In the first analysis of a session, we determine the best fitting relief in the least squares sense. In order to judge the internal consistency of the data, we determine the variations in spatial attitude that remain unexplained by the relief, the so-called "mismatches". These mismatches cannot be integrated to any coherent surface. Thus their magnitude is an indication for the very existence of a "pictorial relief". We show results for one observer (JK), one stimulus (Stimulus I), and one session (Session 1). These are entirely typical (see Fig. 7).

The slant values range up to $70^{\circ}$. This is specific for the example; in many cases we encounter values up to about $90^{\circ}$. The mismatches are peaked near a value of about $10^{\circ}$, which is typical for all observers. There is no doubt that the distributions of the mismatches (in red) and of all slant values (in blue) are very different, indicating that the slants represent meaningful indicators of 3-D structure. The corresponding relief is shown in Fig. $8 .^{3}$

Thus, a first conclusion is that the drawings elicit true volumetric impressions. They are not merely experienced as planar designs. The relief is rather articulate. The arm comes forwards, the leg turns backwards. Bulges corresponding to breast and buttocks are clearly apparent.

The relief should be compared with the drawing (Fig. 4, left), and the mismatch map (Fig. 7, right). The drawing is remarkable in that views of the body are represented that could never be seen simultaneously in an actual pose. This involves a strenuous twist between the thoracic and the pelvic regions. One indeed finds an increased level of mismatch at the region of transition. Other regions of increased mismatch have to do with complications of the contour, such as T-junctions or ending inner contours. They occur near armpit, breast, and buttocks area.

When multiple sessions are available we also determine the spread in the observed spatial attitudes. We find that the mismatches found in the fits of the relief are satisfactorily accounted for by the spread in repeated observations. ${ }^{4}$ This is an issue that we have considered in more detail in

\footnotetext{
3 Although results over observers and stimuli are qualitatively, and semiquantitatively, similar, a detailed analysis reveals some significant variations. Since these play hardly any role in the analysis offered in the bulk of the paper, we mention them in this note. The main differences are between stimuli, observers being very similar. The median depth ranges and interquartile intervals encountered are $177\{158,194\}$ for Stimulus I, 156 $\{149,165\}$ for Stimulus II, and $267\{258,319\}$ for Stimulus III. The median and interquartile range for the discrepancies are $6.86\{6.29,7.55\}$ for Stimulus I, $7.55\{7.12,8.82\}$ for Stimulus II, and $14.74\{13.92,17.19\}$ for Stimulus III. It may be concluded that Stimulus III (indeed in many respects the most extreme drawing) is special as compared to Stimuli I and II.

${ }^{4}$ In order to check this we use the fitted surface in a Monte Carlo simulation. We perturb each normal and refit a surface. This yields the r.m.s. depth mismatch for that level of perturbation. In multiple sessions we find the spread in observed normals, thus we can immediately test whether this spread explains the observed depth mismatches.
} 

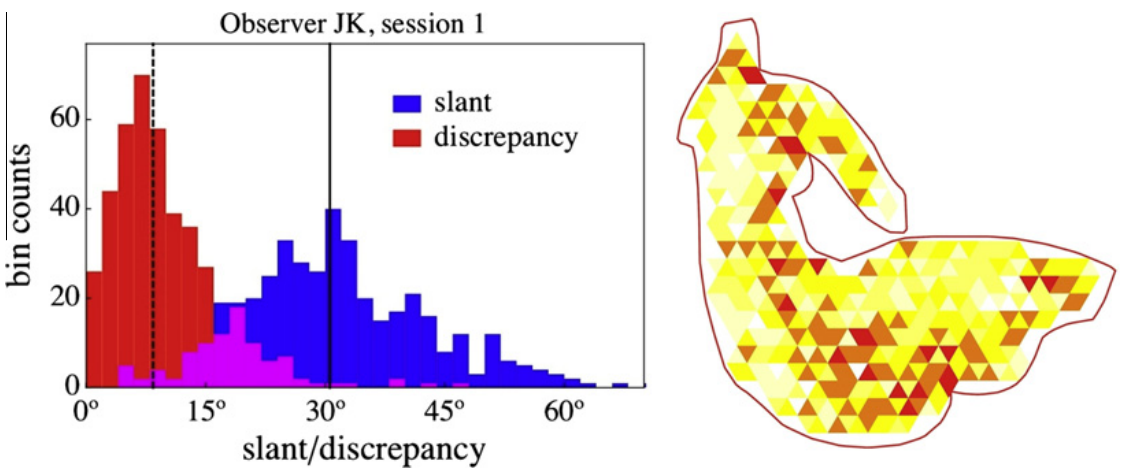

Fig. 7. Left: A histogram of the mismatches (in red) and of all slant values (in blue) for the case of Stimulus I (the overlap region tinted magenta), Observer JK, Session 1. This is representative for all cases. The dashed black line indicates the median of the mismatches, the drawn black line the median of the slants. Right: A topographical map of the distribution of mismatches. The scale runs from white (zero), over yellow, to red (maximum value).
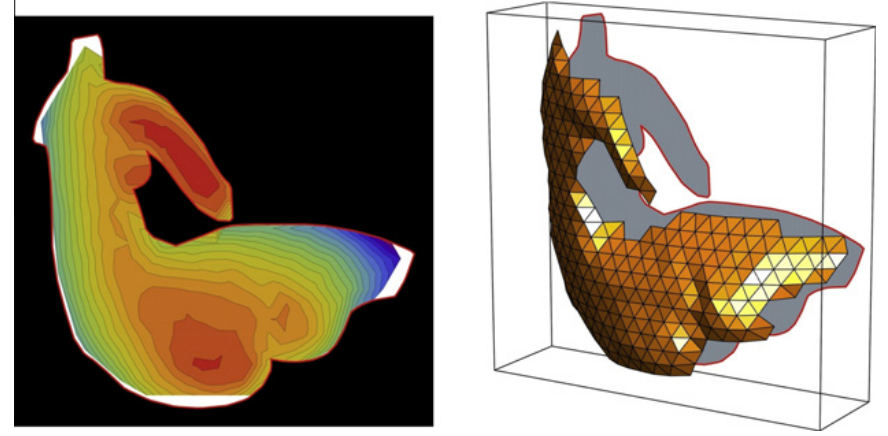

Fig. 8. Left: A contour plot of the depth of relief. Right: The relief has been plotted in pictorial space. The back panel is the picture plane, the region sampled in the measurement is indicated on it. The orthogonal dimension represents depth. Case of Stimulus I, Observer JK, Session 1.
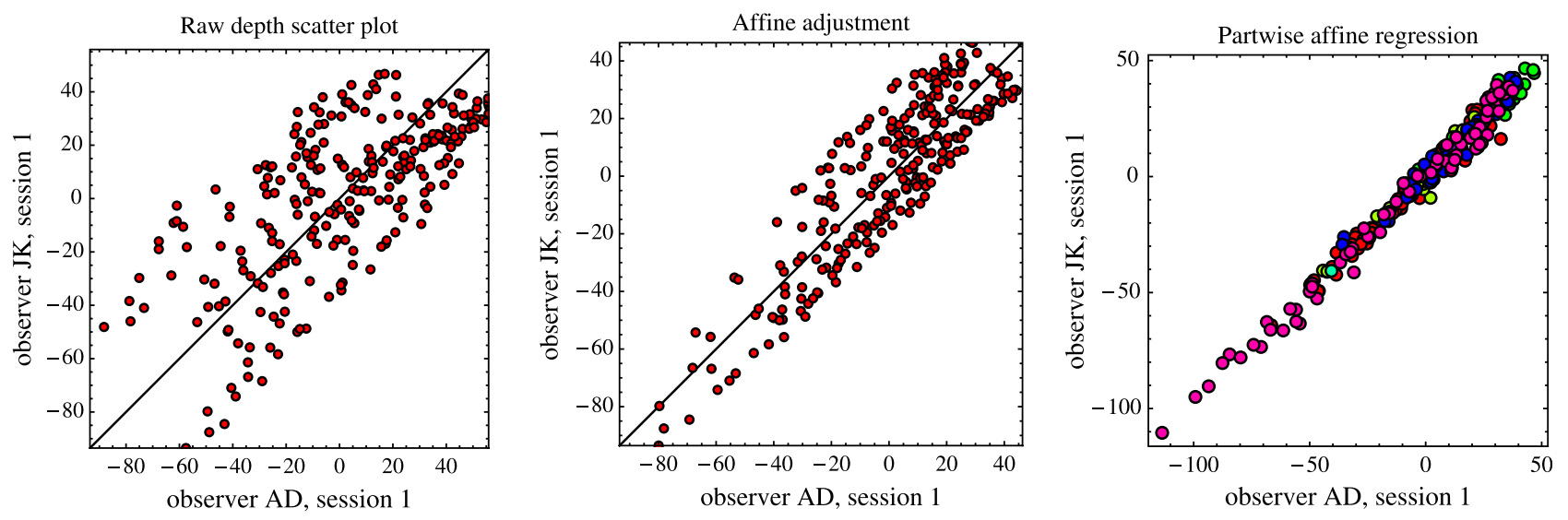

Fig. 9. Scatter plots of the depth values at corresponding locations for Stimulus I, Observers AD and JK, Sessions 1. Left: A comparison of the raw depths $\left(R^{2}=0.49\right)$. Center: A comparison of the globally affinely corrected depths $\left(R^{2}=0.81\right)$. Right: A comparison after a part-wise affine correction $\left(R^{2}=0.95\right)$. The colors in the right-hand figure correspond to those used in Fig. 13, right.

previous studies (Koenderink et al., 1992). It indicates that the pictorial relief is indeed significantly non-planar.

From a comparison of the total depth range and the spread in repeated observations, we find that the observer distinguishes many depth layers. Thus, the relief is known quite accurately from these observations.

\subsection{Session comparisons}

In order to discuss the comparison of different sessions, either by the same or by two different observers (it makes no formal difference), we consider an example into some detail. The comparisons for the other cases are performed in the same way. The main comparison involves the reliefs. In cases of doubt we also check the mismatch maps.

Since we compare the reliefs for a single stimulus, it is natural to compare the depth values at corresponding points. In Fig. 9 (left) we show a scatter plot for the example. This plot compares the depths at corresponding points for the first sessions of observers AD and JK, for Stimulus I. The corresponding depth maps are shown in Fig. 10. The differences are apparently systematic. 
The coefficient of determination of the data in Fig. 9 (left) is only meager (just below one half), so the initial impression may well be that the reliefs are very different. This impression would be too hasty though. In Fig. 9 (center) we show the result of a multiple correlation involving the picture plane coordinates. The fitted relation is $z_{2}=A+R_{x}$ $x+R_{y} y+G z_{1}$, where $A$ is a depth shift (irrelevant), $R_{x}$, and $R_{y}$ are the coefficients of a shear (or non-Euclidean rotation), and $G$ is a scaling factor. The parameters $z_{1}$ and $z_{2}$ denote the depths at corresponding points in the two reliefs.

This result is familiar from numerous previous experiments (e.g., Koenderink \& van Doorn, 2003). Observers often skew the apparent fronto-parallel plane and apply depth dilations or contractions. In this case the apparent fronto-parallels are mutually skewed by less than $10^{\circ}$, whereas there is a depth range shrinkage of a few percent. Notice that the scatter plot does in no way resemble normally distributed scatter, apparently there exists even more hidden structure. We explore it below.

The regional dependency becomes visible if we plot the deviation from the regression line in Fig. 9 center as a function of position. This is shown in Fig. 11. It is immediately obvious that the deviations are not at all randomly distributed, but occur in a structured pattern. This is something we encounter again and again in such comparisons. It is obviously important, and will be discussed in more detail below.

Another way to study the regional dependence is the following. We determine the gauges in a small neighborhood of every vertex (the six neighboring vertices and the vertex itself), thus we again determine the relation $z_{2}=A+R_{x} \quad x+R_{y} \quad y+G \quad z_{1}$, where $A$ is a depth shift (irrelevant), $R_{x}$, and $R_{y}$ are the coefficients of a shear (or non-Euclidean rotation), and $G$ is a scaling factor. The parameters $z_{1}$ and $z_{2}$ denote the depths at corresponding points of the reliefs. We characterize the local gauge by the triple $\left\{R x, R_{y}, G\right\}$, discarding the irrelevant depth shift $A$. The result is shown in Fig. 12. Notice that these variations look all but random. They are apparently connected with the structure of the drawing.

There are various ways to make the nature of this pattern explicit. One way we have implemented is the following. We map the relief into the $\left\{R_{x}, R_{y}, G\right\}$-parameter space. In this space we perform a cluster analysis, using the $k$ means algorithm (MacQueen, 1967; Steinhaus, 1957). One may use various values for $k$ and pick the lowest one that is considered to explain the data. In practice we use

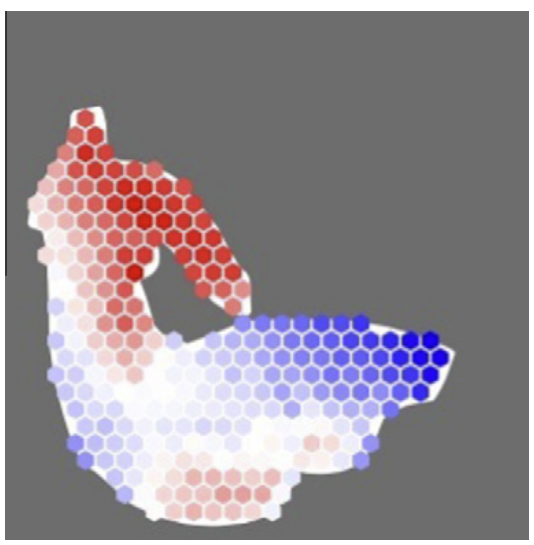

Fig. 11. The regional dependence of the deviations from the regression line in Fig. 9. Red denotes positive, blue negative deviations. Case of Stimulus I, Observers AD and JK, Sessions 1.

only values of two or three. The clusters (Fig. 13, left) may be mapped back onto the corresponding positions in the picture plane. It is not necessarily the case that the clusters map on connected regions, of course. If they do not then we simply split them into connected regions. In the example we set $k=3$. They are not singly connected (Fig. 13, center). After splitting we come up with nine connected regions (Fig. 13, right; four of the sub-regions are so small that they might as well be integrated into one of the larger ones). An affine correction per sub-region (all nine) yields the scatter plot as shown Fig. 9 (right).

The regions identified by the cluster analysis apparently correspond to "natural body parts" and relate to the pronounced twist between the thoracic and pelvic regions. Some of the smaller regions correspond to singularities of the contour (neck, breast). Notice that the concept of "natural body part" is used in the sense common in the art of figure drawing, which is unlike its daily life counterpart. For instance, it is artistically important to count the knee sometimes to the lower, sometimes to the upper leg, depending upon the pose (see the discussion in Hogarth, 1970). We explore the relations with contour singularities later in this paper.

\subsection{Overall results}

In this section we consider the results using a global view, encompassing all observers and all sessions. The analysis is the same for all three stimuli. In order to avoid
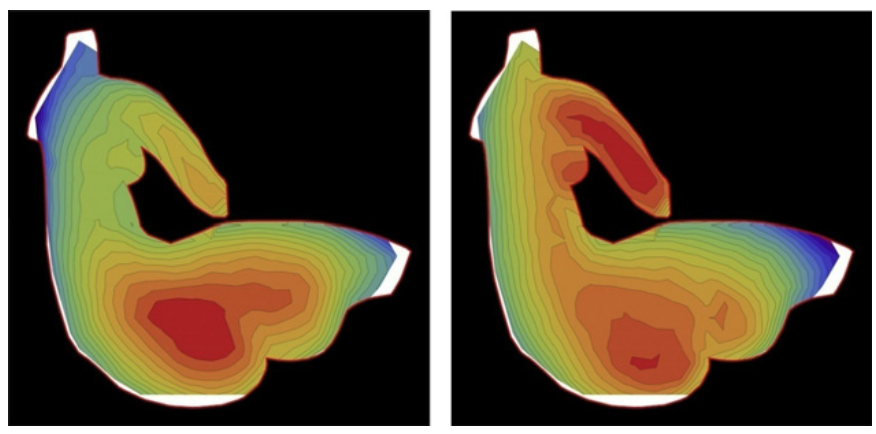

Fig. 10. Depth maps for Stimulus I, Observers AD and JK, Sessions 1. 


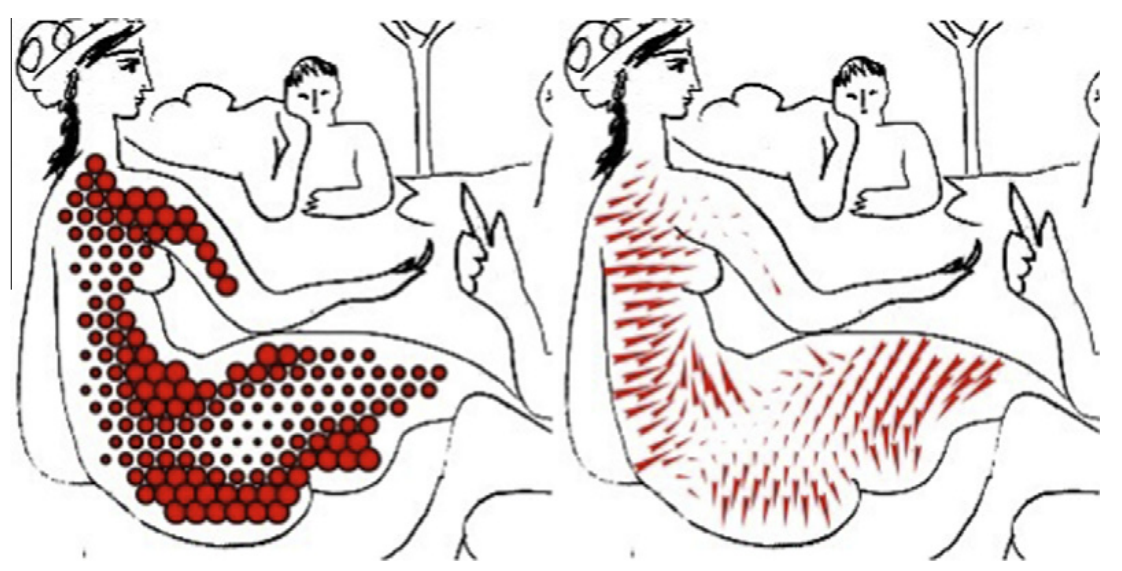

Fig. 12. Regional variations discovered by fitting local gauge transforms. Left: The local scalings (diameter encodes magnitude). Right: The local shears (the arrows show the depth gradient of the local apparent frontal plane). Case of Stimulus I, Observers AD and JK, Sessions 1.
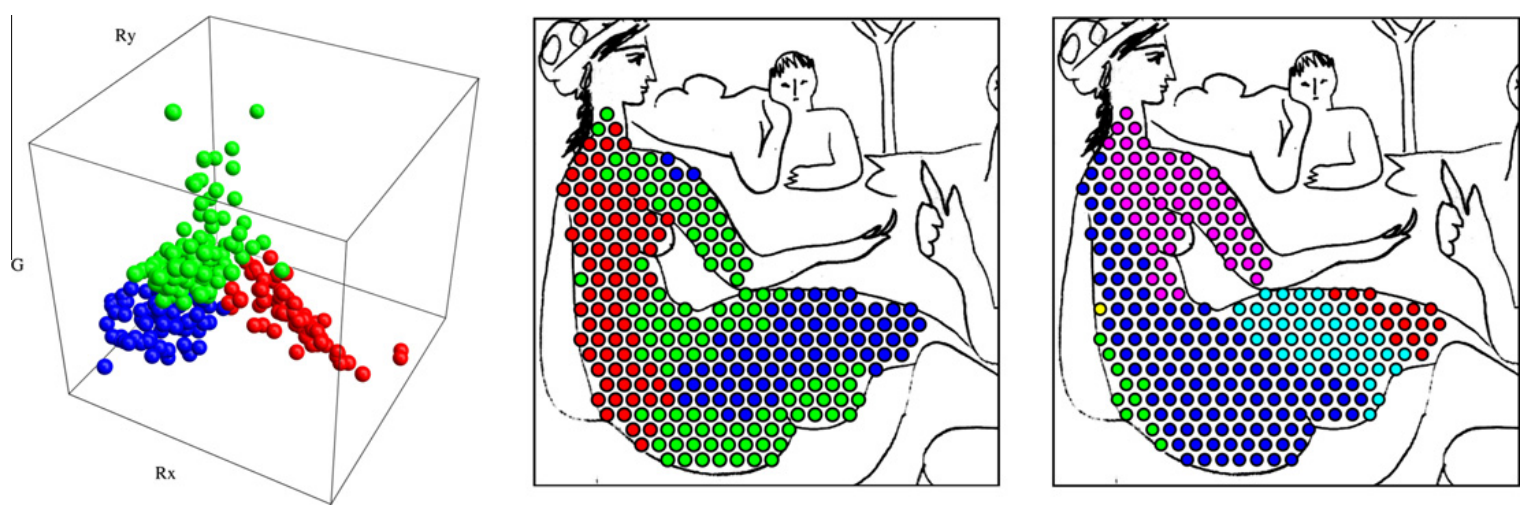

Fig. 13. Left: The clusters found by the clustering algorithm. Center: The regions corresponding to the clusters. The colors of the points in the clusters at left correspond to the colors of the dots in the regions at center. Right: The regions obtained by splitting the non-connected regions at center into the largest singly connected parts. The colors of the dots making up the parts correspond to the colors of the dots in the scatter plot shown in Fig. 9 (right). Case of Stimulus I, Observers AD and JK, Sessions 1.

repeats we discuss the case of Stimulus I in some detail, mentioning the cases of Stimuli II and III more summarily. Most of the remarks apply equally to all three cases with only minor quantitative differences. When there are qualitative or major quantitative differences we report these.

\subsubsection{Grand mean depth}

As a first step we calculate the overall grand mean of the depths. These are illustrated in Fig. 14. Although these are averages over 13 instances, the articulation of the relief is very clear. Apparently the individual differences are sufficiently small that the major features of the relief are well preserved in the mean. Several parts are seen to protrude (buttocks, breast, arm), whereas others recede (neck, leg) into depth. ${ }^{5}$

Given the grand mean, we find the affine transformations that best move the individual results towards the mean. These adjustments serve to remove the effects of individual depth scalings and changes in the apparent

\footnotetext{
${ }^{5}$ Notice (again) that in this paper we use coarse anatomical terms to indicate regions in the picture. This is done for convenience, otherwise we would need to use Cartesian coordinate ranges for instance. It is perhaps not superfluous to mention that no actual anatomical parts are in the picture. It is even unlikely that Picasso used a model in doing this drawing.
}

fronto-parallel planes. This is a desirable step in the comparisons, as has been found to be the case on numerous occasions (Cornelis et al., 2003, 2009; Koenderink \& van Doorn, 2003; Koenderink et al., 2001). It is a necessity that is also well understood in formal terms (Koenderink \& van Doorn, 2008). In the final instance this derives from the intrinsic ambiguities of the pictorial cues (Berkeley, 1709). ${ }^{6}$

We find a range of scalings such that the extremes differ by a little over ten percent. This is a rather narrow range in view of earlier findings. The apparent frontoparallel planes differ by up to $6^{\circ}$, again small variations as compared to earlier findings. In the past we have encountered depth scalings differing by up to a factor of four, and changes of the apparent frontal planes up to $60^{\circ}$ (Koenderink \& van Doorn, 2003). We have no principled way to understand why the deviations are so minor in this case. One possibility is that the reliefs are experienced as roughly frontal, whereas we frequently

\footnotetext{
${ }^{6}$ Notice that we except the so called physiological cues here (Graham, 1965; Helmholtz, 1856). Examples of physiological cues are accommodation and defocus blur. That is why we differentiate between "pictorial cues" and "monocular cues." These differ, among more, by the physiological cues.
} 

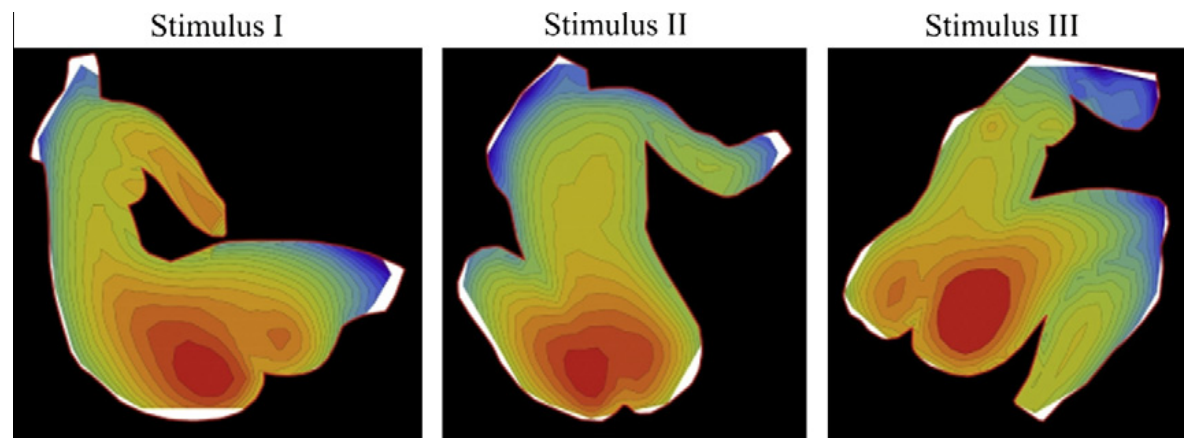

Fig. 14. The overall grand mean of the depths. The colors are a "temperature scale" for nearness, with blue indicating the greatest, red the least depth. Thus (at left, for Stimulus I) the buttocks, the arm, and-to a less extent-the breast protrude, whereas the neck and leg recede into depth. (Notice that these figures have been cropped. Compare with Fig. 5 for clarity.).

included significantly slanted surfaces in previous work (Koenderink \& van Doorn, 2003; Koenderink et al., 2001).

A measure of concordance are the coefficients of determination of the raw depths and of the affinely adjusted depths with the grand mean. This is shown in Fig. 15, left and center columns. Apparently, the various reliefs are all quite similar to the grand mean.

\subsubsection{Deviations from the grand mean depth}

The differences of the individual depth maps with the grand mean are of immediate interest as they reveal the nature of the variation in the responses. Plots of the raw differences between sessions reveal interesting patterns. A straight principal components analysis (PCA) suggests that about five components suffice to explain threequarters of the variance in all cases (Fig. 16). Unfortunately, PCA yields only linear combinations of intuitively reasonable factors (in case there are any), rendering interpretation difficult. One desires a method that yields more natural results.

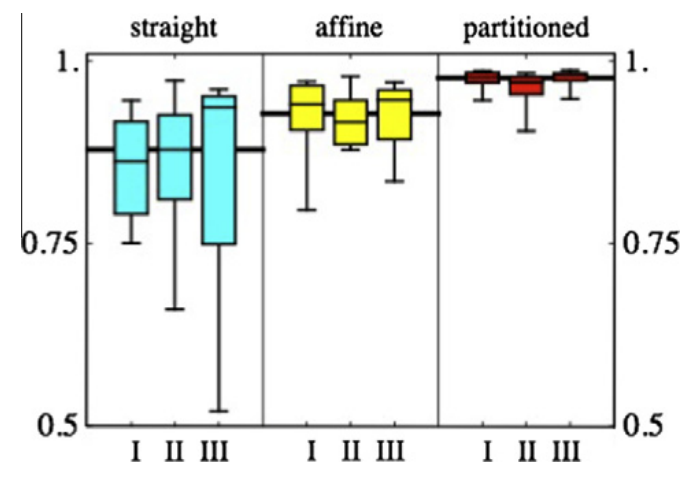

Fig. 15. The ranges of coefficients of determination $\left(R^{2}\right)$ of all sessions of all observers (total of 13 instances) regressed against the grand mean of the depth relief. The results are plotted for the individual Stimuli I, II and III. The thick horizontal lines denote the overall median values (all sessions, all observers, all stimuli). In the left column the regressions of the raw depths against the grand mean, in the center column the regressions including a global affine correction, and in the right column a regression based on partial affine corrections. In the latter case, we used the overall partitions as shown in Fig. 17. Notice that the affine adjustment (center) makes a major difference when compared with the raw depths (left). After partitioning (Fig. 17), the coefficients of determination become quite high (right).
A convenient way to do this is to partition the relief into "natural districts" (Maxwell, 1870). Notice that any (smooth) landscape can be divided into either hills or dales. Here we use the partition into "hills", a hill being defined as a bulge that protrudes towards the observer. Thus, a "summit" is a near point of the relief. If one starts at some point and follows the path that leads towards the observer, one will end up at some summit. The point is then assigned to the hill area that belongs to this summit. In this way any point of the relief is assigned to some unique summit.

The partitions we find for the reliefs of the individual observers and stimuli have a median number of 5 hills (interquartile range 4-7), with a minimum of 2 and a maximum of 11. This suggests that we have a similar "resolution" as with PCA, though in a much more intuitive format.

In order to assess the efficaciousness of partitioning to account for the data, we defined a single overall partition for each stimulus. Each such partition conforms as closely as possible to the individual partitions of the individual observers. The method used to arrive at these partitions, and measure of fit (the "adjusted Rand index"), are explained in Appendix A. These overall partitions turn out to yield a convenient summary of the structure in the data.

After this overall partitioning, all reliefs conform closely to the grand mean (Fig. 15, right). Here we did independent affine adjustments for each partition. The partitioning yields an obvious increase in concordance between observers as compared with mere affine adjustment. A plausible inference is that the observers differ on a piece-wise basis.

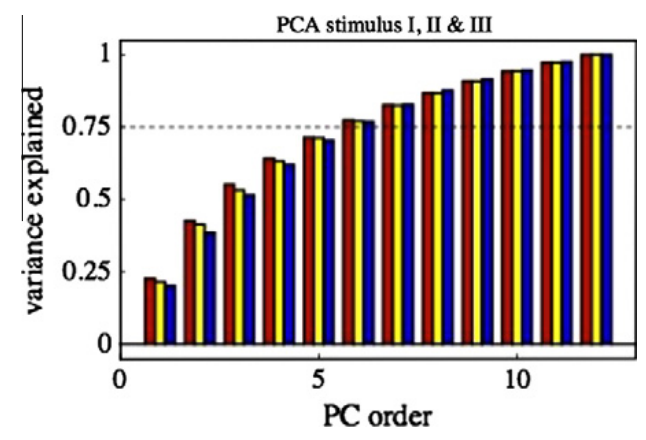

Fig. 16. The fraction of variance explained as a function of PC order in a PCA for Stimulus I (red), II (yellow) and III (blue) involving all observers and all sessions. 

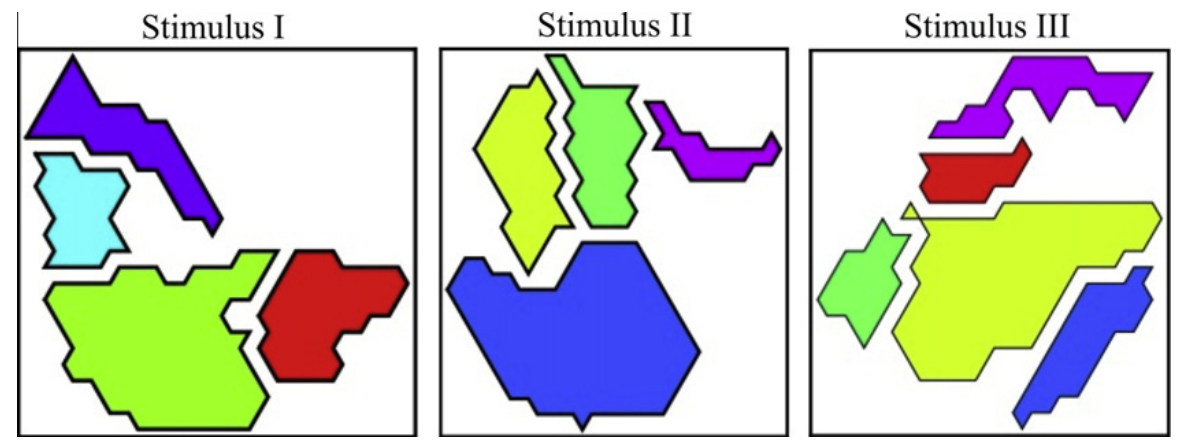

Fig. 17. The overall partitions for Stimulus I, II, and III. These partitions have high median adjusted Rand correlations (see Appendix A) with the raw partitions of all observers and all sessions. (Notice that these figures have been cropped. Compare with Fig. 5 for clarity.).

The overall partition in hills apparently agrees well with the partitions we encountered in the analysis of the pairwise comparison of sessions (Fig. 13). The boundaries of the parts of the partition also appear to agree with the locations of relatively high mismatch in the construction of the relief from local spatial attitude observations (as Fig. 7, right).

\subsection{Relation to the structure of the drawings}

In the previous section we discussed the empirical data. The major results are well summarized in terms of the mean depth maps (Fig. 14), and the overall partitions of the reliefs into hills (Fig. 17). The grand mean and the overall partition into hills characterize the concordance over the group of observers and sessions. The partitions indicate local areas where observers tend to differ in their visual awareness of the shape.

It is a priori likely that these structures of visual awareness might be understood in terms of the geometrical structure of the drawing. Given the general structure of these drawings one might especially look for possible correlations with the singularities or remarkable points of the contour, such as contour terminals, T-junctions and extrema of contour curvature, as well as with global structural properties due to the pairing of opposite stretches of contour, such as medial axes and centers.

We again primarily discuss the case of Stimulus I, summarily commenting on the other cases when these illustrate a certain point particularly well. The three stimuli are similar in most respects, although some features may be expressed somewhat more explicitly in one than in the others.

We first discuss the analysis of the structure of the stimuli, then proceed to put this structure into connection with the observed results. A final summary of conclusions will be presented in the discussion section.

The individual strokes of the drawings carry intrinsic meaning (apart from their roles in the context of the figure as a whole), as illustrated in Fig. 18. We analyze them separately and report the crucial features.

\subsubsection{Analysis of stimulus structure}

In the analysis we start with an analysis of the strokes used by Picasso to build the shape of the figure. It is easily

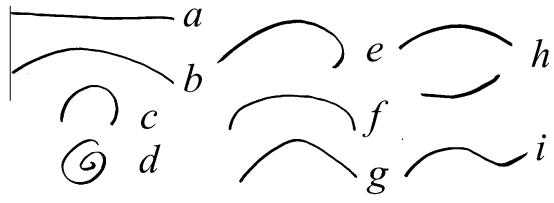

Fig. 18. Some typical strokes. Stroke $a$ is flat and looks clean, mechanical (slightly less objectionable because hand drawn). Stroke $b$ has (roughly) constant curvature and looks boring (this would be worse if drawn by mechanical means). Stroke $c$ is seen to outline a solid volume, whereas stroke $b$ (not enough directional range), and $d$ (too much directional range) do not. Stroke $e$ is a "generic simple stroke" in that it is curved in one sense, with curvature gradually changing uniformly along the stroke (Coxeter, 1989). Strokes $f$ and $g$ show strokes with curvature extrema. Stroke $f$ looks "flattened," stroke $g$ looks differently according to whether it is seen as convex (it looks like a protrusion) or concave (it appears to separate two parts). The two strokes at $h$ evidently belong together and define an ovoid (volumetric) region. Finally, stroke $i$ changes quality along its course, this is Hogarth's (1773) "variety", the key property of his "line of beauty".

visible in the reproductions where the artist lifted the drawing instrument. Picasso is very economical in the use of lines, the figure in Stimulus I is drawn using only eight strokes (see Fig. 19). Some of these more or less stand alone (like stroke 1 defining the breast), others need to be considered as pairs (for example strokes 7 and 8 together defining the arm). Strokes may belong to more than one of such pairs, for instance, stroke 8 defines the arm in connection with stroke 7 , and the shoulder girdle (topmost part of the trunk) in connection with stroke 3. Stroke 2 is the only really complicated one; most are smoothly curved in a single sense (strokes $1,3,4$, and 5), or have a single inflection (stroke 6). Stroke 2 was apparently drawn in one motion, but is actually a compound of three parts, standing for the thoracic region, the belly, and the hipleg region. This is apparent from the pairing of one part of stroke 2 with stroke 6 , another part with stroke 4 .

The strokes were sampled and approximated through analytic curves (the curves shown in Fig. 19, right are actually such analytic curves). ${ }^{7}$ This allows us to analyze the curves using straight differential geometry. (Of course, this

\footnotetext{
${ }^{7}$ We used linear combinations of Chebyshev polynomials in either Cartesian or polar coordinates, using the lowest order of approximation that reproduced the drawn shapes within the accuracy implied by the line thickness. For Stimulus I (Fig. 19 right) powers ranged from 1 (the neck stroke 5 is linear) to 32 (stroke 2).
} 

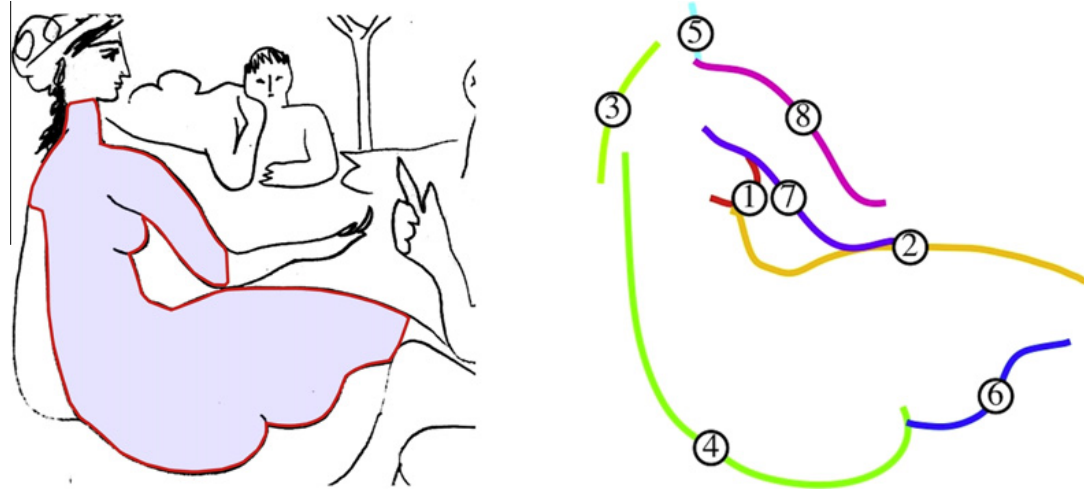

Fig. 19. The individual strokes $1 \ldots 8$ that make up the major figure in Stimulus I. These are the fitted, analytical curves, though these are almost indistinguishable from the actual sets of black pixels in the image.
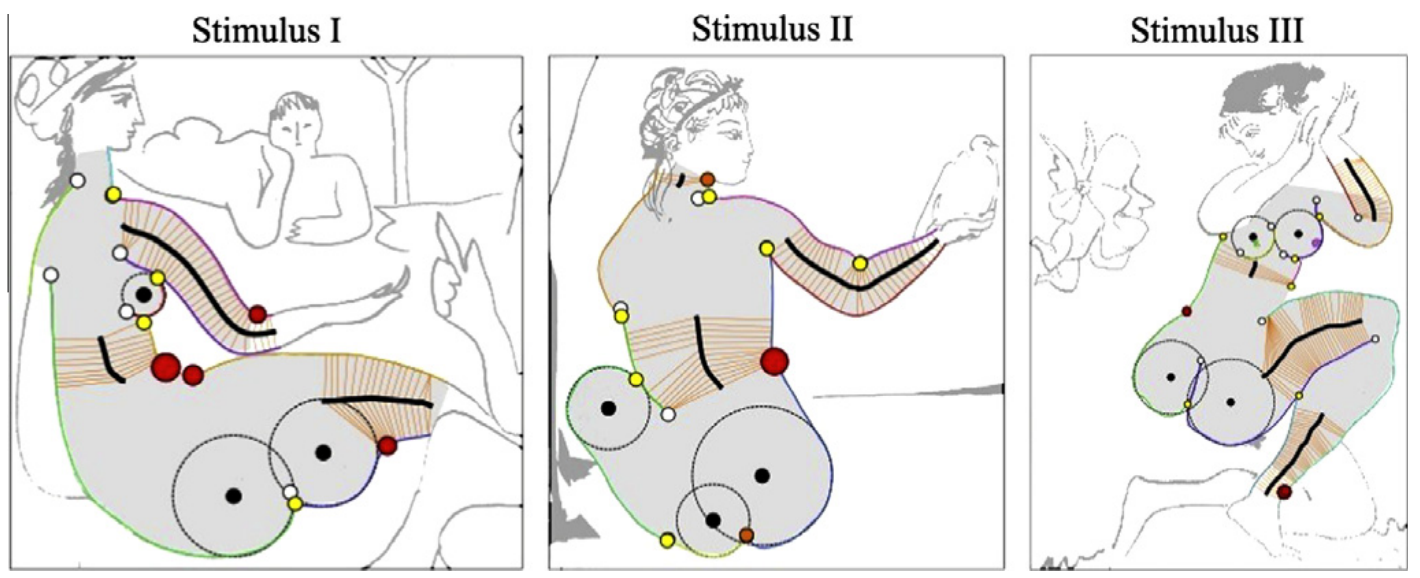

Fig. 20. The individual strokes that make up the major figure in Stimulus I, II and III, augmented with medial axes (thick black lines), centers (thick black points), extrema of negative curvature (red disks, diameter indicates degree of curvature), contour terminals (white circles), and T-junctions (yellow circles). Singular hook singularities in the buttocks and chin areas of Stimulus II are shown as the orange dots.

means that we had to ignore the variations in thickness.) In Fig. 20, the locations and strengths of maximum curvature of concave regions were found by this analysis.

The relations between the strokes are important in eliciting the desired visual effect, and Picasso indicated them carefully. It is easy to identify the contour terminals and T-junctions by close scrutiny of the drawing. They are indicated in Fig. 20. Notice that not all stroke end-points are "contour terminals", for instance (in the case of Stimulus I), stroke 6 has no contour terminal, whereas stroke 4 has contour terminals at both ends. Notice also that the T-junction on stroke 6 that is due to the front-most figure does not count for the analysis because it does not serve to define the shape of the fiducial figure. It is simply ignored in the visual analysis of this figure.

We used the pairings of strokes $\{7,8\},\{4$ (part),2(part)\}, and $\{2$ (part), 6 (part) $\}$ to construct medial axes (example for Stimulus I, the other drawings were treated similarly). When only parts of strokes are used the limits are immediately evident from the structure of the resulting medial axis. The drawn axes in Fig. 20 are of maximum extent, taking more of any of the two strokes introduces awkward hooks.

In some cases a stroke apparently bounds a highly curved convexity, or ovoid shape. In such cases the curve implies a "center". The centers can be approximated by fitting a circle to either the whole stroke (like stroke 1 , the breast), or part of a stroke (strokes 4 and 6 , the buttocks). These centers are also indicated in Fig. 20.

In the case of Stimulus II, there also exist two sharp "hooks" that have been indicated by orange ${ }^{8}$ dots in Fig. 20. Such hooks can be understood as the coincidence of a T-junction with an ending contour (see below).

Another relevant analysis of the drawing involves the "distance transform" of the contour (Blum, 1973). It is shown in Fig. 21. In order to find the distance transform one computes the shortest Euclidean distance to the contour (any point) from a point in the interior of the contour. Thus, one obtains such a minimum distance for any point of the interior. This field of distances obviously takes the value zero on the contour, and reaches a maximum at one or more points inside the contour. For instance, the distance transform for a circle has a single maximum at the center, there the minimum distance equals the radius. If one plots the minimum distance along a dimension orthogonal to the picture plane one obtains a surface (for the example of the circle it is a cone). The distance trans-

\footnotetext{
${ }^{8}$ For interpretation of color in Figs. 5, 7, 8, 9, 11, 13, 14, 16, 20, 21, A, the reader is referred to the web version of this article.
} 

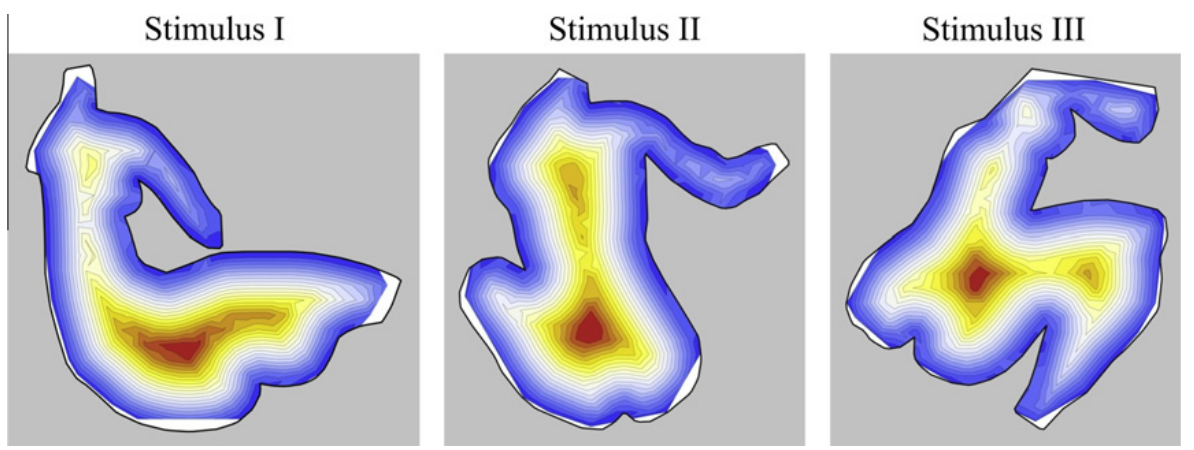

Fig. 21. The distance transform of the contours of Stimulus I, II and III. The color scale is a "temperature scale" for nearness. Thus, the contour is blue (as deep as one gets), whereas the yellowish, and reddish regions are nearest. (Notice that these figures have been cropped. Compare with Fig. 5 for clarity.).

form yields a non-planar surface with a planar outline (the zero distance on the contour). This surface is due to an algorithm that recognizes only the planar shape of the surface, and is fully ignorant of depth cues such as T-junctions and contour terminals. The natural districts (hill regions) of this surface are shown in Fig. 22.

That the distance transform necessarily produces a planar contour renders it useless as a model of monocular stereopsis. Human observers are keenly aware of the depth articulation of contours (Koenderink, van Doorn, Kappers, \& Todd, 1997). However, the comparison is of interest because the distance transform uses only planar structure. It is sometimes held that the perception of drawings is likewise an inference on the basis of purely planar structure. The comparison shows that this does not apply to our observers.

\subsubsection{Comparison of stimulus structure with observations}

First we remind the reader of the differential geometric relations between the various singularities of the contour (Fig. 23). These represent three generic [a, c, d] and one singular [b] view of the same relief from four different, but adjacent, viewpoints. In [a] one sees two hills divided by a valley. The hills are suggested by the two (left and right of the center) convex arcs, divided by a (central) concave arc. (The two inflection points that divide these sub-arcs of the stroke have not been drawn, but are visually apparent.) The contour can be drawn in one stroke, without lifting the drawing instrument. Remember that a

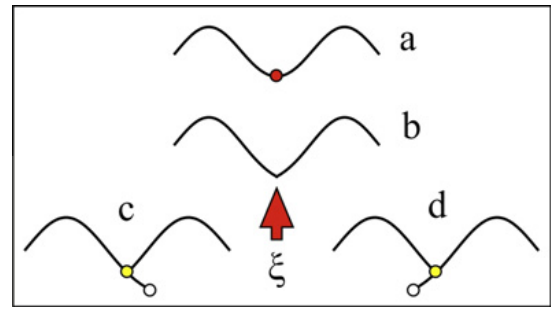

Fig. 23. Various aspects of the contour for diverse (but adjacent) views of a single relief. In (a) the contour is smooth. One sees two hills due to the presence of a maximum of concavity. In (c and d) one sees two hills, one in front of the other. This involves the juxtaposition of a contour terminal and a T-junction. In (b) one has the (singular) transition case, the sharp kink in the contour at $\xi$ is potentially either an extreme concavity, or a \{contour terminal, T-junction\}-pair.

convex contour indicates an elliptic area, necessarily convex (thus a "hill"), whereas a concave contour indicates a hyperbolic (or saddle-like) area, a "pass" (Koenderink \& van Doorn, 1982). The hills appear object-like, the pass their connection. At the deepest point of the pass one has an extremum of concave curvature. Such extrema have been identified as the boundaries of "natural parts" (De Winter \& Wagemans, 2006; Hoffman \& Richards, 1984).

This illustrates the potential meaning of these points in Fig. 20. In cases [c] and [d] (Fig. 23) the contour is made up of two distinct strokes (the artist would have to raise the drawing instrument). One has the juxtaposition of a
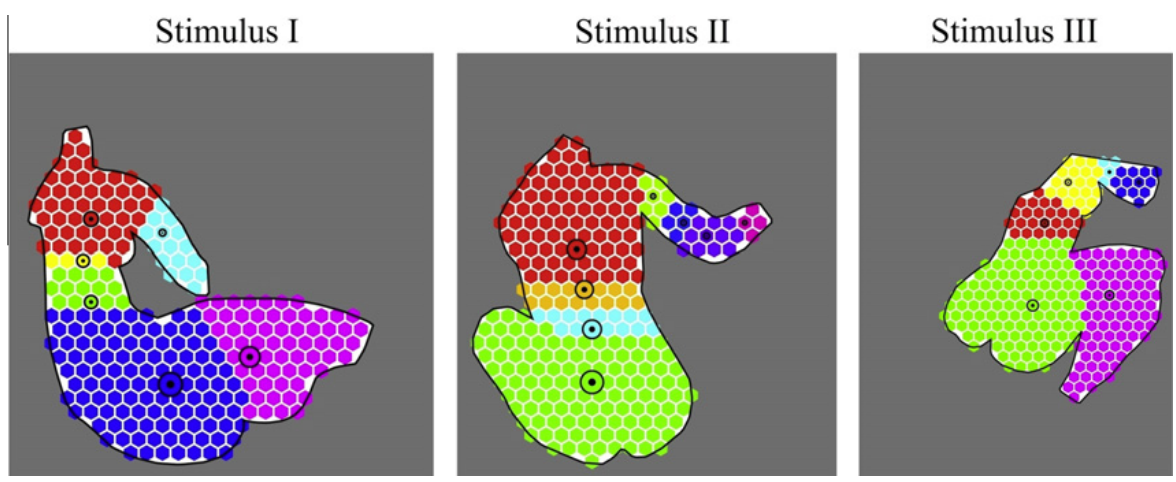

Fig. 22. The natural districts (hill regions) of the distance transform surface (compare Fig. 21) of Stimulus I, II, and III. The summits are indicated by the circles (diameter proportional to their heights). 
contour terminal and a T-junction. Their order along the contour indicates the depth order of the hills (in [c] the left hill is in front, in [d] the right one.) In Fig. 20 one has several instances of such pairs (buttocks, breast, neck). Notice that the extremum of negative curvature and the (contour terminal, T-junction\}-pair may transform into each other as one smoothly varies the viewpoint. At the very moment of the transition, one has the view illustrated in Fig. 23 [b]. It is a sharp kink (first order discontinuity) in the contour. This singularity "bundles" the properties of the extremum of negative curvature and the \{contour terminal, T-junction\}-pair. Although it can be drawn as a single stroke, most artists would draw it in two separate ones, often resulting in case [c] or [d].

Examples of hook-like singularities of the contour are found in Stimulus II (Fig. 20 center, the orange points). They help to give the outline a pleasant arabesque appearance. (The hook can be easily interpreted as essentially "planar", whereas a $\{\mathrm{T}$-junction, contour terminal\}-pair almost forces a spatial, 3-D impression.) Nevertheless, sufficient regular depth cues remain to define the volumetric nature of the body.

The surface of the human body is a complicated landscape of elliptic and hyperbolic areas (Gauß, 1827; Hilbert \& Cohn-Vossen, 1932; Koenderink, 1990). Concave elliptic areas are rare (e.g., the navel), whereas convex elliptic areas come in any size. The latter are seen as "object-like", whereas the hyperbolic areas have no individual meaning (e.g., are not generally named as natural body parts) and mainly appear as a kind of "glue" between the objects. This is common knowledge in the art of sculpture (Alberti, 1435; Rogers, 1969). In drawing this is evident in the treatment of both the contour and the shading of the interior (Koenderink, 1990; Koenderink \& van Doorn, 1982). In the Picasso drawings we deal with the contour alone, since the interior is left blank, so the structure of the interior is necessarily provided by the observer.

The grand mean depth maps (Fig. 14), with the overall hill partitions (Fig. 17), clearly reveal the structure of the major "material" (that is on the figure-side of the contour) white spaces. They are readily named in terms of major natural body parts. These parts are also found in the partition into hills of the distance transform (Fig. 22). For the three stimuli the regions induced by the distance transform are very similar to the mean partitions as shown in Fig. 17 (adjusted Rand index 0.71 for Stimulus I, 0.67 for Stimulus II, and 0.34 for Stimulus III; see Appendix A). But notice that the division into hills of the distance transform is a feature that can be fully accounted for on the basis of purely planar structures. (Of course, this in no way implies that the observers ignore depth cues.) The coefficients of determination for a regression of the distance transform with the individual (affinely adjusted) depth maps are rather low though. The reason is the specific structure of the distance transform.

The distance transform has a singular shape in that it has a planar outline. This is unlike the observed reliefs. For instance, the relief for the grand mean of Stimulus II (Fig. 14, center) has a twisted shape with a markedly non-planar outline. This cannot be accounted for in terms of mere planar features, it involves an awareness of the relief in 3-D pictorial space. In fact, none of the observed reliefs has anything close to a planar outline.

Stimulus III is special because of its use of omitted contour (Fig. 4, right). Notice that the head is not at all connected to the body, ${ }^{9}$ the neck, right shoulder (left and right with respect to the picture surface!) and entire top of the right upper arm are lacking. So are the lower contour of an upper leg, and the belly part of the outline of the thoracic area on the right. Thus, the contour terminal of the corresponding stroke is not a singularity of any "projection". This is common enough though, for instance, the endings of the strokes corresponding to breasts are rarely concave, as they should be geometrically (Koenderink \& van Doorn, 1982).

The strokes that make up the contour, especially the singularities of the contour (Fig. 20) and the partitions in hill regions of the distance transform (Fig. 22) may be expected to suffice to account for the observations of the relief. This is indeed the case to an appreciable extent. Parts are evidently induced through the extremities of concave curvature, centers and medial axes, whereas the contour terminals and $\mathrm{T}$-junctions largely determine local frontaft relations. However, we also find certain features in the observed reliefs that remain unexplained.

An instance of such unexplained features is shown in Figs. 24 and 25. This is the case of the back area in Stimulus II. Although human backs have considerable articulations due to the spine and scapulæ, such articulations are not evident in the shape of the contour. Any algorithm that is based on purely planar structure, such as the distance transform (Fig. 21, center) cannot explain such structure. There are evidently marked differences between the distance transform and the reliefs. This is a case where we have no option but to assume that observers occasionally use template structures when the available constraints are insufficient to determine a relief. In this particular case observer JK apparently applied a template (spinal rut and scapular protrusions), whereas observer HR apparently did not draw on an expertise in human anatomy. ${ }^{10}$

\section{Discussion}

How the human observer constructs an immediate visual awareness of volumetric shapes on the basis of mere line drawings remains largely a mystery. No algorithm in machine vision succeeds in dealing with a fair sample of line drawings as one might find in the print cabinets of many museums of fine art. ${ }^{11}$ Nor are algorithms successful in producing line drawings of either geometrical representations of objects, or photographs of objects, with any degree of success. ${ }^{12}$ So much for the downside. On the upside, from

\footnotetext{
9 That is to say, visually it is, but there are no actual strokes to "prove" the connection.

${ }^{10}$ A reason might be that observer JK has some experience in drawing the human form, whereas the other observers have not. Observer JK is the only one who shows a well developed spinal depression.

11 Major successes of machine vision are in limited domains. Perhaps the most famous instance is the "blocks world", originally introduced by Terry Winograd (1972).

12 Of course, this ignores restricted areas of obvious success, like architectural, machine or map drawings. Here we define "success" roughly as: "ready to compete on a moderate level in any mainstream art gallery".
} 

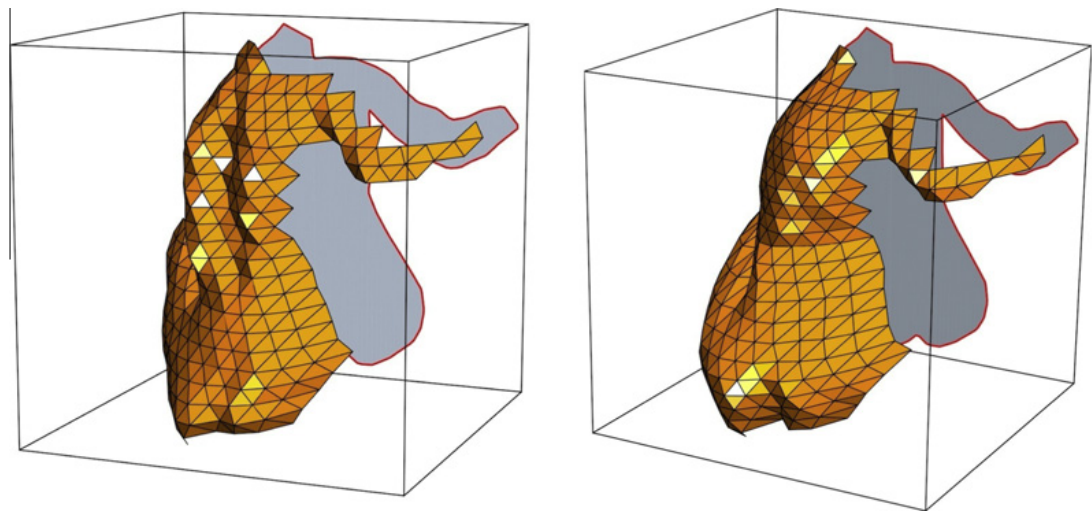

Fig. 24. Reliefs observed by JK (Session 3) and HR (Session 3) for Stimulus II. Notice the pronounced difference in the back. The rut of the relief along the spine is very evident for JK, but totally absent for HR.

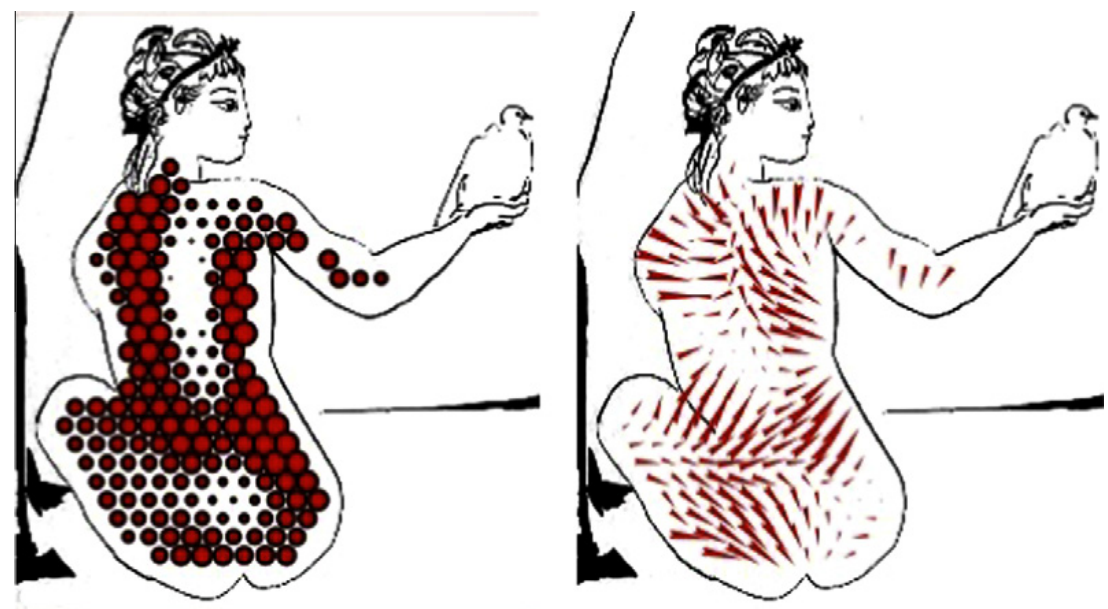

Fig. 25. Local differences between JK Session 3 and HR Session 3 (representation as in Fig. 12). Notice the differences in the thoracic area.

experimental psychology we know quite a bit about local and global depth cues that are doubtlessly used by the human observer, and from musings by artists on their artistic praxis we know quite a bit about the construction of effective drawings.

In this study, we have measured pictorial reliefs for five observers, four of these doing three independent sessions, for three drawings by Pablo Picasso. The drawings were selected because they are obviously representational (they all depict human bodies in various poses), yet by no means "correct", in the sense that they are unlike anything that might be obtained by way of a central projection. (Say by tracing a photograph.) Thus, the drawings might be said to "represent multiple viewpoints", yet they are evidently meant to be appreciated as a unified view. Standard methods based upon a variety of "inverse optics" algorithms are unlikely to find much application here. Yet, human observers are evidently able to build structures in their visual awareness. One naturally wonders whether such structures are similar for different observers, or any single observer at different occasions. One also wonders whether the "depth cues" are effective in the expected ways. Because these depth cues are sparse, we stand a good chance to be able to reach conclusions on this point. Finally, one wonders whether such drawings are primarily enjoyed as planar designs (like the patterns in heraldry or formal embroidery perhaps), or whether they are enjoyed as vehicles towards 3-D pictorial worlds. In the latter case one wonders about the extent of the "observer's share" (Gombrich, 1961). Because the drawings are so sparse (most of the paper remains blank), the observer's share almost has to be appreciable. The drawings serve as weak constraints (certainly as compared to actual scenes, or even straight photographs) on the creative microgenesis of the observers.

We find that the purely planar structure of the drawings is sufficient to account for much, though by no means all of the data. For instance, the distance transform yields a relief that is in many respects very similar to the reliefs we observe in our experiment. The comparison has to be based on the structure of natural districts, or partition into hills. This is because the relief due to the distance transform is a ruled surface of constant slope, that is unlike the smoothly curved reliefs observed in the experiment. The adjusted Rand index of the distance transform partition to the best overall partition from the experiment is very high (see Appendix A). The planar shape thus accounts fully for the partition into hills, yet it uses no "depth cues" at all.

Although the planar structure of the drawings is obviously important, it cannot be concluded that the observers 
are only aware of planar designs. The observed reliefs have structures that are twisted in depth such that their outlines are non-planar. This is impossible to explain from the distance transform (or related algorithms). The microgenesis apparently acts "in depth", and may move an arm forward or backward in pictorial space. Moreover, we notice that the depth cues (contour terminals, T-junctions and contour curvature) have noticeable influence on the observed reliefs. Thus, there is no doubt that both 2-D and 3-D factors play a role in the perception of the drawings.

We also find that neither the purely planar structure, nor the depth cues are sufficient to account for all of the structure encountered in the data. In the drawings most of the paper is left blank, and "perceived" structure in the blank areas is either due to effects from the neighborhood of that area, or has to be supplied by the observer. We find evidence of both cases in the data. For instance, a convexly curved arc whose direction changes steadily over about $60^{\circ}$ or more elicits the notion of an ovoid shape. Examples are breasts and buttocks in the drawings. Here the outline of the blank area leads to a volumetric perception of the (blank!) interior. In other cases we find clear evidence of the application of a template. Such templates may be surprisingly structured. For instance, a clear case in the experiment involves the relief of the human back, including the central, longish groove of the spine and the lateral protrusions of the scapulæ.

That all these influences play a key role in the microgenesis of visual awareness when viewing such drawings may well contribute to their artistic value. Mere planar design is often valued for its formal qualities, but is rarely experienced as exciting. Mere 3-D articulation is exciting (one reason for the popularity of the stereoscope in Victorian households, and perhaps the current stereo hype in Hollywood) but is soon experienced as trivial. The drawings seem to impose about the optimum degree of constraint on our natural microgenesis (see also Van de Cruys \& Wagemans, 2011). It may be the reason why such drawings, that Picasso may have drawn casually over a glass of absinth, remain highly valued on the art market.

In this study, we counted on the ability of our observers to segment the area of the drawing into objects. Any line divides the blank area around it (at least locally) into two parts, an inside ("figure", "stuff", "object", ...), and an outside ("nothing”, "background", “something else”, ...). If you cannot "see" this intuitively, and immediately, there is no way you can parse drawings like these used here. You will see lines as linear distributions of pigment. At this, you would be right (it is the "veridical perception", Denis, 1890) but you could not experience the drawings as "something else" (say a nude, a horse, or a landscape). This is only one example. There are many of such abilities that we silently take for granted, although they are not self evident. It is unlikely that your cat or dog would make much of the drawings. This becomes clear if you ask young children (ones that can just express themselves verbally) to comment on the contents of pictures (Hochberg \& Brooks, 1962). Yet, they are no doubt far ahead of the animals. The present study involves mature persons of contemporary Western upbringing. In Picasso's time many people expressed doubts as to whether his drawings represented anything.
In summary, we have presented evidence that representational (but not necessarily "correct") line drawings may give rise to the awareness of volumetric objects, that are constrained by the drawing, and include a substantial "beholder's share".

\section{Acknowledgements}

This work was supported by the Methusalem program by the Flemish Government (METH/08/02), awarded to Johan Wagemans. We would like to acknowledge administrative support by Stephanie Poot, and useful comments by three reviewers and the editor.

\section{Appendix A. Finding the best overall partitions}

In this appendix we describe the procedure used to arrive at the overall partitions mentioned in the text. Remember that we have to consider 13 different partitions (all sessions, all observers) for each stimulus. We desire to define a single, overall partition for each stimulus in such a way that it reflects the structure in some "best possible" way. Some kind of metric is evidently needed.

A useful measure of the mutual concordance of two partitions is the adjusted Rand index. Remember that the Rand index (after William M. Rand, 1971) is a measure of the concordance of two partitions of a set $S$. Given two partitions $X$ and $Y$, one counts:

$-a$, the number of pairs of elements in $S$ that are in the same subset in $X$ and in the same subset in $Y$,

$-b$, the number of pairs of elements in $S$ that are in different subsets in $X$ and in different subsets in $Y$,

$-c$, the number of pairs of elements in $S$ that are in the same subset in $X$ and in different subsets in $Y$,

$-d$, the number of pairs of elements in $S$ that are in different subsets in $X$ and in the same subset in $Y$.

Then the Rand index is defined as $(a+b) /(a+b+c+d)$. Intuitively this is the number of agreements divided by the sum of the number of agreements and the number of disagreements. The adjusted Rand index (Hubert \& Arabie, 1985; Xuan Vinh, Epps, \& Bailey, 2009) is defined as the Rand index corrected for chance, that is minus its expected value, and is normalized by dividing it by the maximum possible value of the Rand index minus its expected value. This adjusted Rand index (ARI) may possibly take on negative values too, although this did never occur in our data analysis. We consider only values in the range zero to one to be of interest.

Applied to the partition data the ARI is found to vary over a wide range of numbers, with median values around one half (Fig. A). This is partly due to the degree of partitioning, since selective merging of hills may possibly raise the ARI.

In order to arrive at an optimum overall partition, we need to consider all possible partitions of the set of all vertices. This is a very large number. For instance, Stimulus I has 256 vertices. The number of possible partitions of this set (Rota, 1964) is its "Bell number", in this case it is 
approximately $7.25 \times 10^{373}$. Apparently exhaustive search will be out of the question. Unfortunately, we are not aware of a principled method - other than exhaustive search - to arrive at a solution. It appears desirable to cut down on numbers.

An immediate move is to notice that subsets of vertices that belong to a single district for each of the 13 partitions need never be sub-partitioned. Thus we are led to define the "basis" as the partition obtained as the set of all intersections. Such a basis is like the coarsest jigsaw-puzzle from which all 13 partitions may be constructed. All vertices in a subset of the basis are members of a single subset of any partition. Thus, any partition can be specified completely in terms of the basis (for example, 54 subsets in the case of Stimulus I) instead of the vertices (256 in case of Stimulus I), a great convenience in the analysis. In the case of Stimulus I this reduces the number of partitions to $1.8 \times 10^{16}$. Although a significant improvement, this is still a huge number.

We notice that the basis for all partitions contains many parts (Fig. B), and that the subsets of vertices that make up the basis are of various sizes. Many of them are really small, and unlikely to be of much relevance. Intuitively, they are considered "noise". For instance, the basis for stimulus I has 54 subsets, but 22 of these contain only a single vertex, whereas the largest subset contains as many as 45 vertices. All subsets with cardinality less than 5 or so (38 of these) are likely to be spurious. This cuts down the number to about $10^{10}$, again a very significant improvement.

As this number of possible partitions is still a huge number, further constraints are desirable. A very important constraint is topological. Evidently, the partitions should be composed of connected subsets of vertices. This further reduces the number of possible partitions by many orders of magnitude. Unfortunately, the exact number is hard to estimate because it depends upon the precise topology. The general combinatorial problem remains unsolved, even for simple cases like covering a chessboard with polyominoes (Golomb, 1994). From the scattered literature on the combinatorial properties of planar tilings (Rhoads,

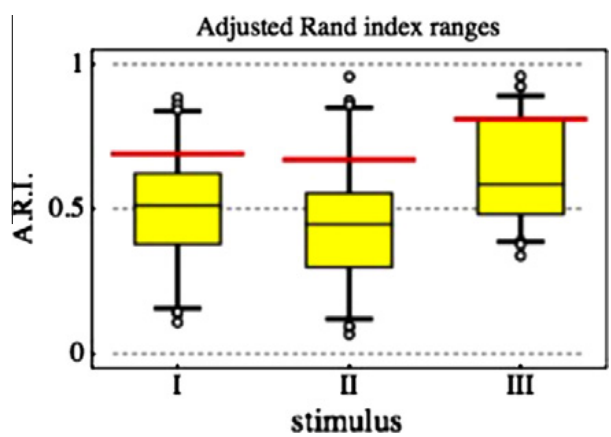

Fig. A. The range of adjusted Rand indices for pairwise comparison of all partitions (each stimulus, all observers, all sessions). The box whisker plots show the quartiles (yellow box), 5\% and 95\% quantiles (whiskers), and outliers (open circles). The red levels indicate the median of the adjusted Rand indices of the correlation between the individual partitions with the overall partitions (shown in Fig. 17). It is apparent that the overall partitions indeed capture most of the common structure very well.
2005) it is clear that the number of possible partitions will be quite large. In any case, an exhaustive search for an optimum is not a viable proposition.

An overall partition (composed of parts of the basis) may be defined as one whose median ARI over all 13 instances is a maximum. Such an optimum, or at least an approximation to it, cannot be found through exhaustive search in reasonable time. In order to arrive at realistic estimates, we designed a stochastic algorithm based on the method of "harmony search" (Geem, Kim, \& Loganathan, 2001). The algorithm applies stratified sampling to sample the whole space at a reasonable resolution. In practice the algorithm allows us to find good approximations in realistic computing times.

The method depends mainly on an algorithm that constructs random partitions of any given complexity. Here "complexity" is simply measured by the number of parts. The highest complexity equals the length of the basis, the lowest complexity is obtained for the trivial partition composed of a single part. These partitions are topologically constrained, so as to produce only connected parts. This can be implemented because the connectivity of the parts is explicitly known. It is a priori likely that the solution will be a partition composed of only a few parts. However, the exact number of parts is unknown. Stratified sampling is implemented by generating simple partitions with higher probability than complex ones, using a Poisson process. A further boost is obtained by generating samples with parts of roughly similar sizes, skipping those with parts composed of a single, or only a few vertices.

The method starts by constructing a number ( $N$ say, some number like 100) of random partitions. It finds the median ARI of each and then divides the set into two subsets of size $M$ (say, something like $N / 4$ will do fine) and $N-M$. The first set is composed of the best samples found thus far, whereas the other set contains the remainder.

The method continues to update the sets indefinitely. In each iteration the set containing the remainder is simply replaced with $N-M$ fresh samples. The set containing the $M$ best samples is updated by slightly perturbing these samples. The perturbation algorithm is simple, it merely (randomly) selects two adjoining regions and (randomly) transfers a part of the basis from one region to the other. If the perturbed sample proves to be better than the original, it replaces it, otherwise the original is kept. After such resampling and perturbation all the $N$ resulting samples are evaluated, and the division into "best samples so far" and "remainder" updated. In some cases a random "hit" may occasionally yield a novel sample with obvious potential, this will change the set of $M$ best samples significantly.

The "current result" is defined as the best sample overall. Repeating the iteration thus yields a sequence of current results. The median ARI of the current results cannot decrease. Typically, it increases by sudden jumps (as the random sampling encounters a "hit"), or by minor increments (as the random perturbations encounter slightly better versions of existing candidates).

The only algorithms that have to be implemented in order to get this iterative procedure going are: 

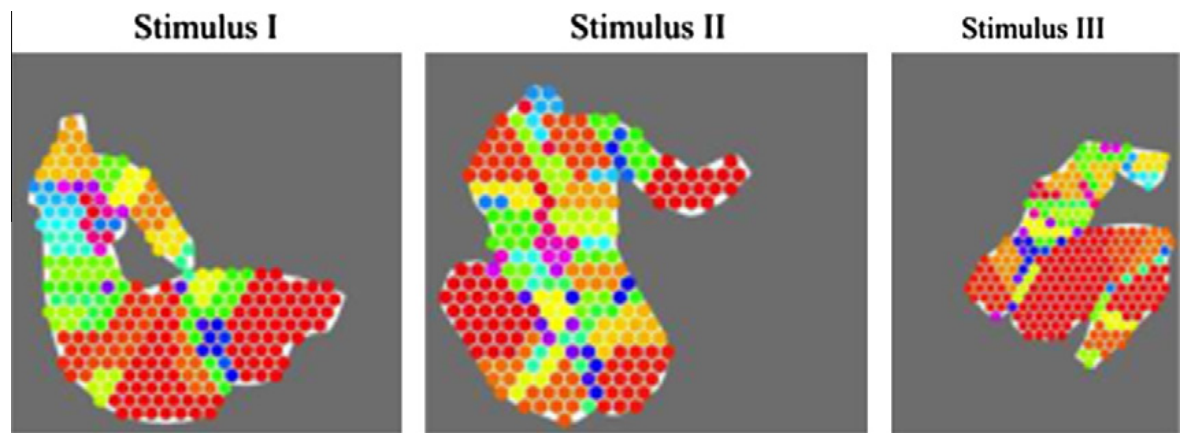

Fig. B. The basis for the partition of reliefs into hill areas for Stimulus I, II and III. Many of the parts are really small, perhaps only a single face of the triangulation. As a realistic estimate of the complexity of these partitions one may count the number of parts needed to cover at least $90 \%$ of the area. We find that one needs 32 parts in the case of Stimulus I, 47 for Stimulus II, and 36 for Stimulus III.

- generating a random partition of given complexity,

- generating a minor random perturbation of a given partition,

- finding the quality of a given partition.

In addition, an overall algorithm "orchestrating" these computations has to be supplied. This overall method contains a few intuitively obvious "tuning" parameters (like $N$, $M$, and the parameter of the Poisson process). None of these methods is complicated, and a large variety of implementations will yield the same result, albeit in different time. For instance, it is evidently possible to turn the method into an exhaustive search by setting $M=0$ and generating random partitions through "fair" sampling. This would render the method useless. On the other hand, even "reasonable" tuning is likely to yield nontrivial results for realistic computing efforts. The method is a very robust one.

This updating is repeated indefinitely. As a stop criterion we simply used a fixed limit (like a thousand updates). This number has been chosen because we find empirically that the current result typically tends to stabilize within the context of such a series. Most often, little happens after a 100 , or 200 iterations. In the initial period there are prolonged series of hardly any change, followed by a sudden jump, and a monotonic increase of the median ARI. The sudden events happen as the algorithm encounters (purely by chance!) a better region in the space of possibilities, the gradual increases after the events are due to the finecombed search in the immediate environment of the "hit".

Notice that the algorithm generates numerous random partitions, even if the current result is already quite good. This ensures that it will eventually find the global optimum (perhaps after zillions of updates) instead of being indefinitely stuck in the neighborhood of some sub-optimum. The perturbation of the currently best samples ensures that the algorithm explores regions that are likely to contain an optimum in small steps, and is thus unlikely to fail to notice the optimum ("jump over it") when close.

In order to sample the space you necessarily have to jump around quite a bit. This could easily render the method ineffective, because you can only sample an almost negligible small part of the space of possibilities. This is effectively counteracted by the stratified sampling, that is by generating "likely" partitions with much higher probability than unlikely ones, and exploring their environments.
We estimate that in generating the end result the algorithm effectively samples about a hundred-thousand likely partitions, in the process implicitly discarding millions of unlikely ones in the sample generation algorithm. This takes about an hour on a $3 \mathrm{GHz}$ Intel Core 2 Duo processor using a Mathematica implementation. A simple method to judge whether the result is of some value, is to run the method a few times from scratch. We found that rerunning the procedure yielded the same result in all cases, thus strengthening our trust in the result.

The "best partition" found in this way turns out to have four parts for Stimulus I and II, five for Stimulus III (Fig. 17). The median ARI is 0.69 for Stimulus I, 0.67 for Stimulus II, and 0.81 for Stimulus III. In view of the differences between observers and sessions, these overall partitions offer an excellent summary of the data (see Fig. A). It is of little importance whether these partitions represent the "true" optimum (if there is a unique one at all), or not. If not, it will be sufficiently close anyway. Although there might indeed exist multiple solutions, we found no trace of such.

\section{References}

Adams, A. (2011). <www.anseladams.com> [visited December 2011].

Alberti, L. B. (1435). Della Pittura. Book I. (Numerous editions and translations available.).

Arnheim, R. (1974). Art and visual perception: A psychology of the creative eye. Berkeley, CA: University of California Press.

Berger, J. (1989). The success and failure of Picasso. New York: Pantheon Books.

Berkeley, G. (1709). An essay towards a new theory of vision. Dublin, UK: Aaron Rhames.

Blum, H. (1973). Biological shape and visual science. Journal of Theoretical Biology, 38, 205-287.

Bouret, J. (1950). Picasso: Dessins. Paris: Diffusion Française.

Brentano, F. (1874). Psychologie vom empirischen Standpunkt. Leipzig: Verlag von Duncker \& Humblot

Brown, J. W. (1996). Time, Will, and Mental Process. New York: Plenum Press.

Cole, F., Sanik, K., DeCarlo, D., Finkelstein, A., Funkhouser, T., Rusinkiewicz, S., et al. (2009). How well do line drawings depcit shape? ACM Transactions on Graphics, 28, 28.1-28.9.

Cornelis, E. V. K., van Doorn, A. J., \& de Ridder, H. (2003). Mirror-reflecting a picture of an object: what happens to the shape percept? Perception and Psychophysics, 65, 1110-1125.

Cornelis, E. V. K., van Doorn, A. J., \& Wagemans, J. (2009). The effects of mirror reflections and planar rotations of pictures on the shape percept of the depicted object. Perception, 38, 1439-1466.

Coxeter, H. S. M. (1989). Introduction to Geometry. New York: Wiley Classics Library series.

Crane, W. (1900). Line and form. London: G. Bell \& Sons, Ltd. 
De Winter, J., \& Wagemans, J. (2006). Segmentation of object outlines into parts: A large-scale integrative study. Cognition, 99, 275-325.

DeCarlo, D., Finkelstein, A., Rusinkiewicz, S., \& Santella, A. (2003). Suggestive contours for conveying shape. ACM Transactions on Graphics, 22, 848-855.

Denis, M. (1890). Définition du néo-traditionnisme. Art et Critique, 65, 540-542.

Euclid (ca. 300 BC). See: Burton, H. E. (1945). The Optics of Euclid. Journal of the Optical Society of America 35, 357-372.

Forsyth, D. A., \& Ponce, J. (2002). Computer vision: A modern approach. Upper Saddle River, NJ: Prentice Hall.

Gauß, C. F. (1827). Disquisitiones generates circa superficies curvas. Commentationes Societatis Regiae Scientiarum Gottingesis Recentiores Volume VI, 99-146. English translation (1902): General Investigations of Curved Surfaces of 1827 and 1825. Princeton, NJ: Princeton University Library. Translated and edited by J. C. Morehead and A. M. Hiltebeitel.

Geem, Z. W., Kim, J. H., \& Loganathan, G. V. (2001). A new heuristic optimization algorithm: Harmony search. Simulation, 76(2), 60-68.

Gibson, J. J. (1950). The perception of the visual world. Boston: Houghton Mifflin.

Golomb, S. W. (1994). Polyominoes (2nd ed.). Princeton, NJ: Princeton University Press.

Gombrich, E. H. (1961). Art and illusion: A study in the psychology of pictorial representation. Princeton, NJ: Princeton University Press.

Graham, C. H. (1965). Vision and visual perception. Oxford, UK: John Wiley $\&$ Sons Inc.

Gurney, J. (1958). Imaginative realism: How to paint what does not exist. Kansas City: Andrews McMeel Publishing, LLC.

Hamm, J. (1963). Drawing the head and figure. New York: Perigee Trade.

Hanks, K., \& Belliston, L. (1992). DRAW! A visual approach to thinking, learning and communication. Los Altos, CA: Crisp Publications, Inc.

Hilbert, D., \& Cohn-Vossen, S. (1932). Anschauliche geometrie. Berlin: Springer.

Hildebrand, A. (1893). Das Problem der Form in der bildenden Kunst. [The Problem of Form in Painting and Sculpture. Translation by M. Meyer and R. M. Ogden (1945). New York: G. E. Stechert.].

Hochberg, J., \& Brooks, V. (1962). Pictorial recognition as an unlearned ability: a study of one child's performance. American Journal of Psychology, 75, 624-628.

Hoffman, D. (1983). The interpretation of visual illusions. Scientific American, 249(6), 154-162.

Hoffman, D. (1998). Visual intelligence, how we create what we see. New York: Norton \& Company, Inc.

Hoffman, D., \& Richards, W. (1984). Parts of recognition. Cognition, 18, 65-96.

Hogarth, W. (1773). The analysis of beauty. Written with a view of fixing the fluctuating ideas of TASTE. London: J. Reeves.

Hogarth, B. (1970). Dynamic figure drawing. New York: Watson-Guptill. 128-129.

Hubert, L., \& Arabie, P. (1985). Comparing partitions. Journal of Classification, 2(1), 193-218.

Judd, T., Durand, F., \& Adelson, E. (2007). Apparent ridges for line drawing. ACM Transactions on Graphics, 26, 19.1-19.7.

Kandinsky, W. (2002, orig. 1926). Punkt und Linie zu Fläche. Beitrag zur Analyse der malerischen Elemente. 8. Auflage. Bern: Benteli.

Kennedy, J. M., \& Silver, J. (1974). The surrogate functions of lines in visual perception: Evidence from antipodal rock and cave art sources. Perception, 3, 313-322.

Klee, P. (1956). Notebooks. Beiträge zur bildnerischen Formlehre (vol. II). Basel: Benno Schwab.

Koenderink, J. J. (1990). Solid shape. Cambridge, MA: MIT Press.

Koenderink, J. J., \& van Doorn, A. J. (in press). Gauge fields in pictorial space. International Journal of Image and Graphics.

Koenderink, J. J., \& van Doorn, A. J. (1982). The shape of smooth objects and the way contours end. Perception, 11, 129-137.

Koenderink, J. J., \& van Doorn, A. J. (1995). Relief: Pictorial and otherwise. Image and Vision Computing, 13(5), 321-334.

Koenderink, J. J., \& van Doorn, A. J. (1998). The structure of relief. Advances in Imaging and Electron Physics, 103, 65-150.

Koenderink, J. J., \& van Doorn, A. J. (2008). The structure of visual spaces. Journal of Mathematical Imaging and Vision, 31, 171-187.
Koenderink, J. J., van Doorn, A. J., Cristou, C., \& Lappin, J. S. (1996a). Shape constancy in pictorial relief. Perception, 25(2), 155-164.

Koenderink, J. J., \& van Doorn, A. J. (2003). Pictorial space. In H. Hecht, R. Schwartz, \& M. Atherton (Eds.), Looking into pictures: an interdisciplinary approach to pictorial space (pp. 239-300). Cambridge, MA: MIT Press.

Koenderink, J. J., van Doorn, A. J., \& Kappers, A. M. L. (1992). Surface perception in pictures. Perception and Psychophysics, 52, 487-496.

Koenderink, J. J., van Doorn, A. J., \& Kappers, A. M. L. (1996b). Pictorial surface attitude and local depth comparisons. Perception and Psychophysics, 58(2), 163-173.

Koenderink, J. J., van Doorn, A. J., Kappers, A. M. L., \& Todd, J. T. (1997). The visual contour in depth. Perception and Psychophysics, 59(6), 828-838.

Koenderink, J. J., van Doorn, A. J., Kappers, A. M. L., \& Todd, J. T. (2001). Ambiguity and the 'mental eye' in pictorial relief. Perception, 30(4), 431-448.

Köhler, W. (1929). Gestalt psychology. New York: Liveright.

MacQueen, J. B. (1967). Some methods for classification and analysis of multivariate observations. Proceedings of 5th Berkeley symposium on mathematical statistics and probability (Vol. 1, pp. 281-297). Berkeley, CA: University of California Press.

Marr, D. (1982). Vision: A computational investigation into the human representation and processing of visual information. New York: Freeman.

Maurer, D., Pathman, T., \& Mondloch, C. J. (2006). The shape of boubas: Sound-shape correspondences in toddlers and adults. Developmental Science, 9(3), 316-322.

Maxwell, J. C. (1870). On hills and dales. Philosophical Magazine, 1870(December), 233-240.

Mellem, J. (2009). Sketching people. Life drawing basics. Cincinnati, OH: North Light Books.

Neurath, O. (1936). International picture language; the first rules of isotype. London: K. Paul, Trench, Trubner \& Co, Ltd.

Pirenne, M. H. (1970). Optics, painting and photography. Cambridge, UK: University Press.

Poggio, T. (1984). Low-level Vision as Inverse Optics. In M. Rauk (Ed.), Proceedings of symposium on computational models of hearing and vision (pp. 123-127), Academy of Sciences of the Estonian S.S.R.

Rand, W. M. (1971). Objective criteria for the evaluation of clustering methods. Journal of the American Statistical Association, 66(336), 846-850.

Rhoads, G. C. (2005). Planar tilings by polyominoes, polyhexes, and polyiamonds. Journal of Computational and Applied Mathematics, $174(2), 329-353$

Rogers, L. R. (1969). Sculpture, the appreciation of the arts/2. London: Oxford University Press.

Rota, G.-C. (1964). The number of partitions of a set. The American Mathematical Monthly, 71(5), 498-504.

Speed, H. (1917). The practice and science of drawing. London: Seeley, Service \& Co, Ltd.

Steinberg, S. (2011). <http://www.saulsteinbergfoundation.org/> [visited December 2011].

Steinhaus, H. (1957). Sur la division des corps matériels en parties. Bulletin Académique Polonais des Sciences, 4(12), 801-804 [in French]

Stevens, K. A. (1981). The visual interpretation of surface contours. Artificial Intelligence, 17, 47-73.

Thom, R. (1973). Stabilité structurelle et morphogenese. New York: Benjamin.

Van de Cruys, S., \& Wagemans, J. (2011). Putting reward in art: A tentative prediction error account of visual art. i-Perception (special issue on Art and Perception), 2(9), 1035-1062. doi: 10.1068/i0466aap.

von Helmholtz, H. (1856). Handbuch der physiologischen Optik (1st ed.). Leipzig: Leopold Voss.

Weston, E. (2011). <www.edward-weston.com> [visited December 2011]

Winograd, T. (1972). Understanding natural language. New York: Academic Press.

Xuan Vinh, N., Epps, J., \& Bailey, J. (2009). Information theoretic measures for clustering comparison: Is a correction for chance necessary? In ICML '09: Proceedings of the 26th annual international conference on machine learning (pp. 1073-1080), ACM 